\title{
Reducible problem for a class of almost-periodic non-linear Hamiltonian systems
}

\author{
Muhammad Afzal ${ }^{1 *}$, Tariq Ismaeel ${ }^{2}$ and Muhammad Jamal ${ }^{3}$
}

\author{
"Correspondence: \\ afzalmuhammad@stu.ouc.edu.cn \\ mafzalmughal700@yahoo.com \\ 'School of Mathematical Sciences, \\ Ocean University of China, Qingdao, \\ P.R. China \\ Full list of author information is \\ available at the end of the article
}

\begin{abstract}
This paper studies the reducibility of almost-periodic Hamiltonian systems with small perturbation near the equilibrium which is described by the following Hamiltonian system:

$$
\left.\frac{d x}{d t}=\right\lrcorner[A+\varepsilon Q(t, \varepsilon)] x+\varepsilon g(t, \varepsilon)+h(x, t, \varepsilon)
$$

It is proved that, under some non-resonant conditions, non-degeneracy conditions, the suitable hypothesis of analyticity and for the sufficiently small $\varepsilon$, the system can be reduced to a constant coefficients system with an equilibrium by means of an almost-periodic symplectic transformation.
\end{abstract}

Keywords: Almost-periodic matrix; Reducibility; KAM iteration; Hamiltonian systems; Small divisors

\section{Introduction}

In this paper we are studying the reducibility of the following almost-periodic Hamiltonian system:

$$
\frac{d x}{d t}=J[A+\varepsilon Q(t, \varepsilon)] x+\varepsilon g(t, \varepsilon)+h(x, t, \varepsilon), \quad x \in \mathbf{R}^{2 N}
$$

where $J$ is an anti-symmetric symplectic matrix, $A$ is a $2 N \times 2 N$ symmetric constant matrix with possible multiple eigenvalues, and $Q(t)$ is an analytic almost-periodic symmetric $2 N \times 2 N$ matrix with respect to $t, g(t, \varepsilon)$ and $h(x, t, \varepsilon)$ are almost-periodic $2 N$-dimensional vector-valued functions with respect to $t$, with basic frequencies $\omega=\left(\omega_{1}, \omega_{2}, \ldots\right)$ and $h(x, t)=O\left(x^{2}\right)(x \rightarrow 0)$, and

$$
J=\left(\begin{array}{cc}
0 & I_{N} \\
-I_{N} & 0
\end{array}\right)
$$

where $I_{N}$ is a $N \times N$ identity matrix and $\varepsilon$ is a sufficiently small parameter. First of all we will recall some previous results in the field of reducibility for analytic differential systems.

(c) The Author(s) 2018. This article is distributed under the terms of the Creative Commons Attribution 4.0 International License (http://creativecommons.org/licenses/by/4.0/), which permits unrestricted use, distribution, and reproduction in any medium, provided you give appropriate credit to the original author(s) and the source, provide a link to the Creative Commons license, and indicate if changes were made. 
Consider the differential equation

$$
\frac{d x}{d t}=A(t) x, \quad x \in \mathbf{R}^{m},
$$

where $A(t)$ is an almost-periodic matrix. We call the transformation $x=P(t) y$ almostperiodic Lyapunov-Perron (L-P) transformation, if $P(t)$ is non-singular and $P, P^{-1}$, and $\dot{P}$ are almost periodic. The transformed equation is

$$
\frac{d y}{d t}=C(t) y
$$

where $C=P^{-1}(A P-\dot{P})$. If there exists an almost-periodic L-P transformation such that $C(t)$ is a constant matrix, then we call equation (2) reducible.

In recent years, many researchers have devoted themselves to the study of the reducibility of finite dimensional systems by means of the KAM methods. The well-known Floquet theorem states that every periodic differential equation (2) can be reduced to a constant coefficients differential equation (3) by means of a periodic change of variables with the same period as $A(t)$. But, if $A(t)$ is quasi-periodic (q-p), then there is an example in [1] which illustrates that (2) is irreducible. In 1981, Johnson and Sell [2] showed that if $A(t)$ the quasi-periodic matrix satisfies "full spectrum" conditions, then (2) is reducible. In 1992, Jorba and Simó [3] proved the reducibility result of linear quasi-periodic systems like (5) for the constant matrix $A$ with distinct eigenvalues. In 1999, Xu [4] proved the reducibility result of linear quasi-periodic systems like (5) for the constant matrix $A$ with multiple eigenvalues. In 1996, Jorba and Simó [5] considered the quasi-periodic system

$$
\frac{d x}{d t}=[A+\varepsilon Q(t)] x+\varepsilon g(t)+h(x, t), \quad x \in \mathbf{R}^{m},
$$

where the constant matrix $A$ has distinct eigenvalues. They proved that system (4) is reducible for $\varepsilon \in E$ using the non-resonant conditions and non-degeneracy conditions, where $E$ is the non-empty Cantor subset such that $E \subset\left(0, \varepsilon_{0}\right)$. Instead of quasi-periodic reduction to a constant coefficient linear systems, in 1996, Xu and You [6] proved the reducibility of the linear almost-periodic differential equation

$$
\frac{d x}{d t}=[A+\varepsilon Q(t)] x, \quad x \in \mathbf{R}^{m},
$$

where the constant matrix $A$ has different eigenvalues and $Q(t)$ is an $m \times m$ analytic almost-periodic matrix with frequencies $\omega=\left(\omega_{1}, \omega_{2}, \ldots\right)$. Under some small divisor conditions and for most sufficiently small $\varepsilon$, they proved that system (5) is reducible to the constant coefficient system by an affine almost-periodic transformation. In 2013, Qiu and Li [7] considered the following non-linear almost-periodic differential equation:

$$
\frac{d x}{d t}=[A+\varepsilon a(t)] x^{2 n+1}+h(x, t, \varepsilon)+f(x, t, \varepsilon), \quad x \in \mathbf{R},
$$

where $n \geq 0$ is an integer, $A$ is a positive number, $\varepsilon$ is a small parameter, $h$ is a higher order term, and $f$ is a small perturbation term. They proved that under some suitable conditions and using the KAM method system (6) can be reduced to a suitable normal form with zero 
as an equilibrium point by an affine almost-periodic transformation, so it has an almostperiodic solution near zero.

In 2015, Li et al. [8] considered the following analytic quasi-periodic Hamiltonian system:

$$
\frac{d x}{d t}=[A+\varepsilon Q(t)] x+\varepsilon g(t)+h(x, t), \quad x \in \mathbf{R}^{2 m},
$$

where the constant matrix $A$ has multiple eigenvalues, $Q, g$, and $h$ are quasi-periodic with respect to $t$ and $h=O\left(x^{2}\right)(x \rightarrow 0)$. They proved that by using the non-resonant conditions, non-degeneracy conditions, and a suitable hypothesis of analyticity, the Hamiltonian system (7) can be changed to another Hamiltonian system with an equilibrium by a q-p symplectic transformation.

In this paper we are going to extend the results of [5] to the almost-periodic Hamiltonian system (1).

This paper is organized as follows. In Sect. 2, statement of the main result is given, in Sect. 3 we give some lemmas which are essential for the proof of the main result, in Sect. 4 the first KAM step is given, in Sect. 5 the main result is proved, and finally, in Sect. 6 conclusion of the paper is given.

\subsection{Definitions and notations}

To state our main result, we need some definitions and notations.

Definition 1.1 We say that a function $f$ is a quasi-periodic function of time $t$ with basic frequencies $\omega=\left(\omega_{1}, \omega_{2}, \ldots, \omega_{d}\right)$ if $f(t)=F\left(\theta_{1}, \theta_{2}, \ldots, \theta_{d}\right)$, where $F$ is $2 \pi$ periodic in all its arguments $\theta_{j}=\omega_{j} t$ for $j=1,2, \ldots, d$. $f$ will be called analytic quasi-periodic in a strip of width $\rho$ if $F$ is analytic on $D_{\rho}=\left\{\theta|| \Im \theta_{j} \mid \leq \rho, j=1,2, \ldots, d\right\}$. In this case we denote the norm by $\|f\|_{\rho}=\sum_{k \in \mathbf{Z}^{d}}\left|F_{k}\right| e^{\rho|k|}$. A function $f$ is almost-periodic if $f(t)=\sum_{n=1}^{\infty} f_{n}(t)$, where $f_{n}(t)$ are all quasi-periodic for $n=1,2, \ldots$.

Definition 1.2 Suppose that $A(t)=\left(a_{s j}(t)\right)$ is a quasi-periodic $m \times m$ matrix. If every $a_{s j}(t)$ is analytic on $D_{\rho}$, then we call $A(t)$ analytic on $D_{\rho}$.The norm of $A(t)$ is defined as follows:

$$
\|A(t)\|_{\rho}=m \times \max _{1 \leq s, j \leq r}\left\|a_{s j}(t)\right\|_{\rho} .
$$

If $A$ is a constant matrix, the norm of $A$ is defined as follows:

$$
\|A\|=m \times \max _{1 \leq s, j \leq r}\left|a_{s j}\right| .
$$

Write $B(0, b)=\{x \in \mathbf{C}|| x \mid \leq b\}$ and $\Delta_{b, \rho}=B(0, b) \times D_{\rho}$.

Definition 1.3 Let $h(x, t)$ be real analytic in $x$ and $t$ on $\Delta_{b, \rho}$, and let $h(x, t)$ be quasiperiodic with respect to $t$ with frequency $\omega$. Then $h(x, t)$ can be expanded as a Fourier series as follows:

$$
h(x, t)=\sum_{k \in \mathbf{Z}^{d}} h_{k}(x) e^{i\langle k, \theta\rangle} .
$$


Then

$$
\|h\|_{\Delta_{b, \rho}}=\sum_{k \in \mathbf{Z}^{d}}\left|h_{k}\right|_{b} e^{\rho|k|},
$$

where $h_{k}(x)=\sum_{n=0}^{\infty} h_{n k} x^{n}$ and $\left|h_{k}\right|_{b}=\sup _{x \in B(0, b)} \sum_{n=0}^{\infty}\left|h_{n k}\right||x|^{n}$. It is easy to see that

$$
\left\|h_{1} h_{2}\right\|_{\Delta_{b, \rho}} \leq\left\|h_{1}\right\|_{\Delta_{b, \rho}}\left\|h_{2}\right\|_{\Delta_{b, \rho}} .
$$

The aim of the study is to develop the reducibility for the almost-periodic non-linear Hamiltonian system (1). To take over the difficulty from the infinite frequency which generates the small divisors problem, we need a stronger norm. Inspired by the works of $[4$, 5], and [8], in this paper, we allow $Q, g$, and $h$ to be the classes of almost-periodic matrices. Our study is about the reducibility of almost-periodic Hamiltonian systems to [4] and [8]. So, the usual LP transformation for KAM iteration should not only be almostperiodic but also symplectic, which preserves the Hamiltonian structure. For this purpose, let us introduce "spatial structure", "approximation function", and some related definitions.

Definition 1.4 ([9]) Let $\tau$ be a set of some subsets of the natural number set $\mathbf{N}$. Then $(\tau,[\cdot])$ is called a finite spatial structure in $\mathbf{N}$ if $\tau$ satisfies:

1. $\emptyset \in \tau$;

2. If $\Lambda_{1}, \Lambda_{2} \in \tau$, then $\Lambda_{1} \cup \Lambda_{2} \in \tau$;

3. $\bigcup_{\Lambda \in \tau} \Lambda=\mathbf{N}$,

and $[\cdot]$ is a weight function defined on $\tau$ such that $[\emptyset]=0,\left[\Lambda_{1} \cup \Lambda_{2}\right] \leq\left[\Lambda_{1}\right]+\left[\Lambda_{2}\right]$.

Denote the weight value by $[k]=\inf _{\operatorname{supp} k \subset \Lambda, \Lambda \in \tau}[\Lambda]$. Write $|k|=\sum_{s=1}^{\infty}\left|k_{s}\right|$.

Definition 1.5 ([4]) If $U(t)=\sum_{\Lambda \in \tau} U_{\Lambda}(t)$, where $U_{\Lambda}(t)$ are quasi-periodic matrices with basic frequencies $\omega_{\Lambda}=\left\{\omega_{s} \mid s \in \Lambda\right\}$, then $U(t)$ is known as an almost-periodic matrix with spatial structure $(\tau,[\cdot])$ and basic frequencies $\omega$, which is the maximum subset of $\cup \omega_{\Lambda}$ in the sense of integer modular. Denote the average of $U(t)$ by $\bar{U}$, where

$$
\bar{U}=\lim _{T \rightarrow \infty} \frac{1}{2 T} \int_{-T}^{T} U(t) d t
$$

Let $U(t)=\sum_{\Lambda \in \tau} U_{\Lambda}(t)$, for $z>0, \rho>0$,

$$
\|U(t)\|_{z, \rho}=\sum_{\Lambda \in \tau} e^{z[\Lambda]}\left\|U_{\Lambda}(t)\right\|_{\rho}
$$

is called a weight norm with finite spatial structure $(\tau,[\cdot])$.

Definition $1.6([10]) \Delta$ is called an approximation function if

1. $\Delta:[0, \infty) \rightarrow[1, \infty)$ is an increasing function and it satisfies $\Delta(0)=1$;

2. $\frac{\log \Delta(t)}{t}$ is decreasing on $[0, \infty)$;

3. $\int_{0}^{\infty} \frac{\log \Delta(t)}{t^{2}} d t<\infty$. 
Remark If $\Delta$ is an approximation function, then so is $\Delta^{4}$.

Definition 1.7 Let $h(x, t)=\sum_{\Lambda \in \tau} h_{\Lambda}(x, t)$ with frequency $\omega=\left(\omega_{1}, \omega_{2}, \ldots\right)$ and with finite spatial structure $(\tau,[\cdot])$, for $z>0, \rho>0$,

$$
\|h(x, t)\|_{z, \Delta_{b, \rho}}=\sum_{\Lambda \in \tau} e^{z[\Lambda]}\left\|h_{\Lambda}(x, t)\right\|_{\Delta_{b, \rho}}
$$

is known as the weight norm of $h(x, t)$.

Definition 1.8 A matrix $S$ is said to be symplectic if $S J S^{T}=J$, where $S^{T}$ represents the transpose of $S$ and $J=\left(\begin{array}{cc}0 & I_{N} \\ -I_{N} & 0\end{array}\right)$, where $I_{N}$ is an $N \times N$ identity matrix.

For a map $\psi(t, x): \mathbf{R}^{2 N} \rightarrow \mathbf{R}^{2 N}$, let $\frac{\partial \psi}{\partial x}$ denote the Jacobian of $\psi$ with respect to $x$, that is, $\frac{\partial \psi}{\partial x}=\left(\frac{\partial \psi_{i}}{\partial x_{j}}\right)_{2 N \times 2 N}$.

Definition $1.9 \psi(t, x)$ is symplectic if and only if the Jacobian of $\psi$ with respect to $x$ is symplectic, i.e., $\frac{\partial \psi}{\partial x} J \frac{\partial \psi}{\partial x}^{T}=J$.

Definition 1.10 A matrix $A$ is said to be Hamiltonian if and only if $A=J B$, where $B$ is a symmetric matrix and $J$ is defined as above.

For our problem, the non-resonant conditions will be

$$
\left|\lambda_{s}-\lambda_{j}-\sqrt{-1}\langle k, \omega\rangle\right| \geq \frac{\alpha}{\Delta^{4}(|k|) \Delta^{4}([k])}
$$

for all $1 \leq s \neq j \leq 2 N$ and $k \in \mathbf{Z}^{\mathbf{N}} \backslash\{0\}$, where $\lambda_{1}, \lambda_{2}, \ldots, \lambda_{2 N}$ are the eigenvalues of $J A, \omega$ is the basic frequencies of $Q(t), \Delta(t)$ is an approximation function satisfying $\sum_{k \in \mathbf{Z}^{\mathrm{N}}} \frac{1}{\Delta(|k|) \Delta([k])}<\infty$ and $\alpha$ is a small positive constant. From [4] and [9], we can choose the weight function

$$
[\Lambda]=1+\sum_{s \in \Lambda} \log ^{p}(1+|s|), \quad p>2 .
$$

\section{Statement of the main result}

Theorem 2.1 Suppose that the Hamiltonian system (1) in which JA is a Hamiltonian matrix with possible multiple eigenvalues, $Q(t)=\sum Q_{\Lambda}(t)$ and $g(t)=\sum g_{\Lambda}(t)$ are analytic almost-periodic matrices with respect to ton $D_{\rho}$, and $h(x, t)=\sum h_{\Lambda}(x, t)$ is analytic almostperiodic matrix with respect to tand $x$ on $\Delta_{b, \rho}$ with basic frequencies $\omega=\left(\omega_{1}, \omega_{2}, \ldots\right)$ and has the spatial structure $(\tau,[\cdot])$ which depends continuously on the small parameter $\varepsilon$. Suppose that JA is a $2 N \times 2 N$ matrix with possible multiple eigenvalues $\lambda_{1}, \lambda_{2}, \ldots, \lambda_{2 N}$ that can be diagonalized and the multiplicity of the eigenvalues $\lambda_{s^{\prime}}$ is $r_{s^{\prime}}, s^{\prime}=1,2, \ldots, l$, $r_{1}+r_{2}+\cdots+r_{l}=2 N, \lambda_{s^{\prime}} \neq 0$, and $\lambda_{s^{\prime}} \neq \lambda_{j^{\prime}}$ with $s^{\prime} \neq j^{\prime}$ and $1 \leq s^{\prime}, j^{\prime} \leq l$. Moreover, assume that $h(x, t, \varepsilon)$ is analytic with respect to $x$ on the closed ball $B_{b}(0), h(0, t, \varepsilon)=0$, and $D_{x} h(0, t, \varepsilon)=0$ and $\varepsilon \in\left(0, \varepsilon_{0}\right)$ is a parameter. If

1. There exists $z>0$ such that $\|Q(t)\|_{z, \rho}<\infty,\|g(t)\|_{z, \rho}<\infty$; 
2. (Non-resonant conditions) $\lambda=\left(\lambda_{1}, \lambda_{2}, \ldots, \lambda_{2 N}\right)$ and $\omega=\left(\omega_{1}, \omega_{2}, \ldots\right)$ satisfy

$$
\begin{aligned}
& \left|\lambda_{s}-\sqrt{-1}\langle k, \omega\rangle\right| \geq \frac{\alpha_{0}}{\Delta(|k|) \Delta([k])} \\
& \left|\lambda_{s}-\lambda_{j}-\sqrt{-1}\langle k, \omega\rangle\right| \geq \frac{\alpha_{0}}{\Delta(|k|) \Delta([k])}
\end{aligned}
$$

for all $1 \leq s, j \leq 2 N, k \in \mathbf{Z}^{\mathbf{N}} \backslash\{0\}, \alpha_{0}>0$ is a small constant and $\Delta(t)$ is an approximation function;

3. (Non-degeneracy conditions) Suppose that the unique solution of $\dot{x}=J A x+\varepsilon g(t)$ is denoted by $\underline{x}$. Assume that $J\left(A+\varepsilon \bar{Q}^{*}\right)$ has $2 N$ different eigenvalues $\lambda_{s}^{1}(\varepsilon)$

$(1 \leq s \leq 2 N)$ which satisfy $\left|\lambda_{k_{1}}^{1}(\varepsilon)-\lambda_{k_{2}}^{1}(\varepsilon)\right| \geq 2 \eta \varepsilon>0, \eta_{2} \geq\left|\frac{d\left(\lambda_{k}^{1}(\varepsilon)\right)}{d t}\right| \geq \eta_{1}>0$, and $\eta_{2} \geq\left|\frac{d\left(\lambda_{k_{1}}^{1}(\varepsilon)-\lambda_{k_{2}}^{1}(\varepsilon)\right)}{d t}\right| \geq \eta_{1}>0$, where $k, k_{1}, k_{2}=1,2, \ldots, 2 N, k_{1} \neq k_{2}, \varepsilon \in\left(0, \varepsilon_{0}\right)$, and $\eta$, $\eta_{1}$, and $\eta_{2}$ are positive constants. Let $Q^{*}(t)=Q(t)+\frac{1}{\varepsilon} D_{x} h(\underline{x}, t)$ and $\bar{Q}^{*}$ be the average of $Q^{*}(t)$.

4. $\left\|D_{x x} h(x, t, \varepsilon)\right\|_{z, \Delta_{b, \rho}} \leq K$, where $\varepsilon \in\left(0, \varepsilon_{0}\right)$ and $x \in B_{b}(0)$.

Then there exists a Cantor subset $E \subset\left(0, \varepsilon_{0}\right)$ with positive Lebesgue measure such that the Hamiltonian system (1) is reducible for $\varepsilon \in E$, i.e., there exists an almost-periodic symplectic transformation $x=\psi(t, \varepsilon) y+\varphi(t, \varepsilon)$, where $\psi(t, \varepsilon)$ and $\varphi(t, \varepsilon)$ are almost-periodic with basic frequencies and spatial structure $(\tau,[\cdot])$ as $Q(t)$, which transforms (1) into the Hamiltonian system

$$
\frac{d y}{d t}=A_{*}(\varepsilon)+h_{*}(y, t, \varepsilon)
$$

where $A_{*}(\varepsilon)$ is the constant matrix and $h_{*}(y, t, \varepsilon)$ is of order two in $y$. Furthermore, for small enough $\varepsilon_{0}$, the relative measure of $E$ in $\left(0, \varepsilon_{0}\right)$ is close to 1 .

Remark In general, we suppose that $g(t), Q(t)$, and $h(x, t)$ depend on $\varepsilon$, but for simplicity, in the following we do not represent this dependence.

\section{Some lemmas}

In this section, we will give some results in the form of lemmas which are useful for the proof of Theorem 2.1 .

Lemma 3.1 ([4]) Suppose that $U$ and $R$ are almost-periodic matrices, and they have the same spatial structure and the same frequencies. If $\|U\|_{z, \rho}<+\infty,\|R\|_{z, \rho}<+\infty$, then $U R$ is an almost-periodic matrix and has the same spatial structure and the same frequencies with $U$ and $R$, and

$$
\|U R\|_{z, \rho} \leq\|U\|_{z, \rho}\|R\|_{z, \rho}
$$

Lemma 3.2 ([4]) Suppose that an analytic almost-periodic matrix $U(t)$ has the spatial structure $(\tau,[\cdot])$, and for $z>0, \rho>0,\|U\|_{z, \rho}<+\infty$. Then, for the average of $U(t)$, we have $\|\bar{U}\| \leq\|U\|_{z, \rho}$. 
Lemma 3.3 ([5]) Let $\lambda_{1}^{1}, \lambda_{2}^{1}, \ldots, \lambda_{2 l}^{1}$ be the eigenvalues of $B_{1}$ satisfying $\left|\lambda_{s}^{1}\right|>2 \nu,\left|\lambda_{s}^{1}-\lambda_{j}^{1}\right|>$ $2 v$, and $v>0$ with $s \neq j$. Consider $B_{0}$ to be a matrix such that $B_{0}^{-1} B_{1} B_{0}=\operatorname{diag}\left(\lambda_{1}^{1}, \ldots, \lambda_{2 l}^{1}\right)$. Define $\beta_{0}=\max \left\{\left\|B_{0}\right\|,\left\|B_{0}^{-1}\right\|\right\}$. Let $\varsigma$ be a value such that $0<\varsigma<\frac{2 v}{(6 l-1) \beta_{0}^{2}}$. Then, if JA verifies $\left\|J A-B_{1}\right\|<\varsigma$, the following results hold:

1. If $\lambda_{1}, \lambda_{2}, \ldots, \lambda_{2 l}$ are the eigenvalues of $J A$, we have $\left|\lambda_{s}\right|>v,\left|\lambda_{s}-\lambda_{j}\right|>v, s \neq j$.

2. There exists a non-singular matrix $B$ such that $B^{-1} J A B=\operatorname{diag}\left(\lambda_{1}, \lambda_{2}, \ldots, \lambda_{2 l}\right)$, which satisfies $\|B\|,\left\|B^{-1}\right\| \leq \beta$, where $\beta=2 \beta_{0}$.

Remark Indeed, by Gerschgorin's lemma, the result 1 of Lemma 3.3 can be obtained. In our case, if the constant matrix $A_{0}$ can be diagonalized, then the eigenvalues $\lambda_{s}^{0}$ of $A_{0}$ satisfy $\left|\lambda_{s}^{0}\right| \geq 2 \eta_{3}>0$ with a constant $\eta_{3}, \forall s$, and $A_{n}-A_{0}=O(\varepsilon)$, where $n \geq 1$ represents the $n$th KAM step. So, by Gerschgorin's lemma, we have that, for small enough $\varepsilon$, the eigenvalues $\lambda_{s}^{n}$ of $A_{n}$ satisfy $\left|\lambda_{s}^{n}\right| \geq \eta_{3}>0, \forall s$. In this article, we denote $v=\eta \varepsilon$ and $B_{1}=A_{1}$. Then Lemma 3.3 holds and, moreover, in this article, $\beta_{0}$ is a bounded and positive constant.

Lemma 3.4 Consider the system

$$
\dot{x}=J A x+\varepsilon g(t), \quad x \in \mathbf{R}^{2 N},
$$

where $J A \in B_{\varsigma}\left(A_{1}\right)$ and $\varsigma$ is given by Lemma 3.3. Let the eigenvalues of $J A$ be $\lambda_{s}$, where $\left|\lambda_{s}\right| \geq \eta_{3}>0$ with a constant $\eta_{3}, \forall s$. Moreover, $g(t)=\sum g_{\Lambda}(t)$ is analytic almost-periodic on $D_{\rho}$ with frequencies $\omega=\left(\omega_{1}, \omega_{2}, \ldots\right)$ and has the spatial structure $(\tau,[\cdot])$. Suppose

$$
\left|\lambda_{s}-\sqrt{-1}\langle k, \omega\rangle\right| \geq \frac{\alpha}{\Delta^{4}(|k|) \Delta^{4}([k])}
$$

$\forall k \in \mathbf{Z}^{\mathbf{N}} \backslash\{0\}$, a constant $\alpha>0$ and an approximation function $\Delta(t)$. Let $0<\bar{\rho}<\rho, 0<$ $\bar{z}<z$. So, for equation (11), a unique analytic almost-periodic solution $x(t)$ exists with the same spatial structure and the same frequency as $g(t)$ which satisfies $\|x\|_{z-\bar{z}, \rho-\bar{\rho}} \leq$ $c \varepsilon \frac{\Gamma(\bar{z}) \Gamma(\bar{\rho})}{\alpha}\|g \mid\|_{z, \rho}$, where $\Gamma(\rho)=\sup _{t \geq 0}\left[\Delta^{4}(t) e^{-\rho t}\right.$ and a constant $c>0$.

Proof Making the change of variables $x=B y$ and by defining $h(t)=B^{-1} g(t)$, system (11) can be written as

$$
\dot{y}=D y+\varepsilon h(t), \quad y \in \mathbf{R}^{2 N},
$$

where $D=B^{-1}(J A) B=\operatorname{diag}\left(\lambda_{1}, \lambda_{2}, \ldots, \lambda_{2 N}\right)$. Let

$$
\begin{array}{ll}
y_{\Lambda}=\left(y_{\Lambda}^{s j}\right), & \left(y_{\Lambda k}^{s j}\right)=\sum_{\operatorname{supp} k \subset \Lambda} y_{\Lambda k}^{s j} e^{\sqrt{-1}\langle k, \theta\rangle}, \\
h_{\Lambda}=\left(h_{\Lambda}^{s j}\right), & \left(h_{\Lambda k}^{s j}\right)=\sum_{\operatorname{supp} k \subset \Lambda} h_{\Lambda k}^{s j} e^{\sqrt{-1}\langle k, \theta\rangle},
\end{array}
$$

where, $\theta=\omega t$. 
Substitute these into $\dot{y}_{\Lambda}=D y_{\Lambda}+\varepsilon h_{\Lambda}$, and by equating the coefficients on both sides, we obtain

$$
y_{\Lambda k}^{s j}=\varepsilon \frac{h_{\Lambda k}^{s j}}{\lambda_{s}-\sqrt{-1}\langle k, \omega\rangle} .
$$

So, by equation (12), we get

$$
\begin{aligned}
\left\|y_{\Lambda}^{s j}\right\|_{\rho-\bar{\rho}} & \leq \varepsilon\left(\frac{1}{\eta_{3}}+\sum_{\operatorname{supp} k \subset \Lambda} \frac{\Delta^{4}(|k|) e^{-\bar{\rho}|k|}}{\alpha} \Delta^{4}([k])\right)\left|h_{\Lambda k}^{s j}\right| e^{\rho|k|} \\
& \leq c \varepsilon \frac{\Gamma(\bar{\rho}) \Delta^{4}([\Lambda])}{\alpha}\left\|h_{\Lambda}^{s j}\right\|_{\rho},
\end{aligned}
$$

where $c>0$ is a constant. Thus

$$
\left\|y_{\Lambda}\right\|_{\rho-\bar{\rho}} \leq c \varepsilon \frac{\Gamma(\bar{\rho}) \Delta^{4}([\Lambda])}{\alpha}\left\|h_{\Lambda}\right\|_{\rho} .
$$

Let $y=\sum_{\Lambda \in \tau} y_{\Lambda}$. From Definition 1.2, we have

$$
\begin{aligned}
\|y\|_{z-\bar{z}, \rho-\bar{\rho}} & =\sum_{\Lambda \in \tau}\left\|y_{\Lambda}\right\|_{\rho-\bar{\rho}} e^{(z-\bar{z})[\Lambda]} \\
& \leq c \varepsilon \sum_{\Lambda \in \tau} \frac{\Gamma(\bar{\rho}) \Delta^{4}([\Lambda])}{\alpha}\left\|h_{\Lambda}\right\|_{\rho} e^{z[\Lambda]-\bar{z}[\Lambda]} \\
& \leq c \varepsilon \frac{\Gamma(\bar{\rho}) \Gamma(\bar{z})}{\alpha}\|h\|_{z, \rho} .
\end{aligned}
$$

By Remark of Lemma 3.3, we have $\|B\| .\left\|B^{-1}\right\| \leq c_{0}$, where $c_{0}>0$ is a constant. Then, since $\|h\|_{z, \rho} \leq\left\|B^{-1}\right\|\|g\|_{z, \rho}$ and $\|x\|_{z, \rho} \leq\|B\|\|y\|_{z, \rho}$, we have

$$
\|x\|_{z-\bar{z}, \rho-\bar{\rho}} \leq c \varepsilon \frac{\Gamma(\bar{z}) \Gamma(\bar{\rho})}{\alpha}\|g\|_{z, \rho} .
$$

Lemma 3.5 Let $h: B_{b}(0) \subset \mathbf{R}^{l} \rightarrow \mathbf{R}^{l}$ be a $C^{2}$ function that satisfies $h(0)=0, \frac{d(h(0))}{d x}=0$, $\left\|\frac{d^{2}(h(x))}{d x^{2}}\right\| \leq K, \forall x \in B_{b}(0)$, where $B_{b}(0)$ is a ball centered at 0 and having radius $b$, where $K$ is a constant. Then $\|h(x)\| \leq \frac{K}{2}\|x\|^{2},\left\|\frac{d(h(x))}{d x}\right\| \leq K\|x\|$.

For the proof of Lemma 3.5, see [5].

\section{Lemma 3.6 Let}

$$
\dot{P}=J A P-P J A+Q
$$

where $J A$ is a $2 N \times 2 N$ Hamiltonian matrix and $J A \in B_{5}\left(A_{1}\right)$, the eigenvalues of $A_{1}$ are $\lambda_{1}^{1}, \lambda_{2}^{1}, \ldots, \lambda_{2 N}^{1}$ with $\left|\lambda_{s}^{1}\right|>2 \eta \varepsilon$ and $\left|\lambda_{s}^{1}-\lambda_{j}^{1}\right|>2 \eta \varepsilon$ for $s \neq j$, and $\varsigma$ can be found in Lemma 3.3 . Let $\lambda_{s}, 1 \leq s \leq 2 N$, with $\lambda_{s} \neq 0$ be the eigenvalues of JA. Moreover, $Q(t)=\sum_{\Lambda \in \tau} Q_{\Lambda}(t)$ is an analytic almost-periodic Hamiltonian matrix on $D_{\rho}$ with frequencies $\omega=\left(\omega_{1}, \omega_{2}, \ldots\right)$ and with finite spatial structure $(\tau,[\cdot]) \cdot \bar{Q}=0$, where $\bar{Q}$ is the average of $Q(t)$. Let

$$
\left|\lambda_{s}-\lambda_{j}-\sqrt{-1}\langle k, \omega\rangle\right| \geq \frac{\alpha}{\Delta^{4}(|k|) \Delta^{4}([k])},
$$


$\forall k \in \mathbf{Z}^{\mathbf{N}} \backslash\{0\}, \alpha>0$ is a constant and $\Delta(t)$ is an approximation function, and $\left|\lambda_{k_{1}}-\lambda_{k_{2}}\right| \geq$ $\eta \varepsilon, k_{1} \neq k_{2}$. Let $0<\bar{\rho}<\rho, 0<\bar{z}<z$. Then we have a unique analytic almost-periodic Hamiltonian matrix $P(t)$ with the same spatial structure and the same frequencies as $Q(t)$, which solves equation (14) and satisfies

$$
\|P\|_{z-\bar{z}, \rho-\bar{\rho}} \leq c \frac{\Gamma(\bar{z}) \Gamma(\bar{\rho})}{\alpha}\|Q\|_{z, \rho},
$$

where $\Gamma(\rho)=\sup _{t \geq 0}\left[\Delta^{4}(t) e^{-\rho t}\right.$ and $c>0$ is a constant.

Proof We can suppose that the matrix $B$ is as in Lemma 3.4. Making the setting $P=B V B^{-1}$ and $R=B^{-1} Q B$, equation (14) can be written as

$$
\dot{V}=D V-V D+R
$$

where $D=\operatorname{diag}\left(\lambda_{1}, \lambda_{2}, \ldots, \lambda_{2 N}\right)$. Let

$$
\begin{array}{ll}
R_{\Lambda}=\left(r_{\Lambda}^{s j}\right), & \left(r_{\Lambda k}^{s j}\right)=\sum_{\operatorname{supp} k \subset \Lambda} r_{\Lambda k}^{s j} e^{\sqrt{-1}\langle k, \theta\rangle}, \\
V_{\Lambda}=\left(v_{\Lambda}^{s j}\right), & \left(v_{\Lambda k}^{s j}\right)=\sum_{\operatorname{supp} k \subset \Lambda} v_{\Lambda k}^{s j} e^{\sqrt{-1}\langle k, \theta\rangle},
\end{array}
$$

where $\theta=\omega t$. Substitute these into $\dot{V}_{\Lambda}=D V_{\Lambda}-V_{\Lambda} D+R_{\Lambda}$, and by equating the coefficients on both sides, we have $v_{\Lambda 0}^{s j}=0$; or

$$
v_{\Lambda k}^{s j}=\frac{r_{\Lambda k}^{s j}}{\lambda_{s}-\lambda_{j}-\sqrt{-1}\langle k, \omega\rangle} \quad \text { for } k \neq 0
$$

As $Q$ is analytic on $D_{\rho}$, therefore $R=B^{-1} Q B$ is also analytic on $D_{\rho}$. So, by using equation (15), we have

$$
\begin{aligned}
\left\|v_{\Lambda}^{s j}\right\|_{\rho-\bar{\rho}} & \leq \sum_{\operatorname{supp} k \subset \Lambda} \frac{\Delta^{4}(|k|) e^{-\bar{\rho}|k|}}{\alpha} \Delta^{4}([k])\left|r_{\Lambda k}^{s j}\right| e^{\rho|k|} \\
& \leq \frac{\Gamma(\bar{\rho}) \Delta^{4}([\Lambda])}{\alpha}\left\|r_{\Lambda k}^{s j}\right\|_{\rho} .
\end{aligned}
$$

Thus

$$
\left\|P_{\Lambda}\right\|_{\rho-\bar{\rho}} \leq \frac{\Gamma(\bar{\rho}) \Delta^{4}([\Lambda])}{\alpha}\left\|R_{\Lambda}\right\|_{\rho} .
$$

Let $V=\sum_{\Lambda \in \tau} V_{\Lambda}$. From Definition 1.4, we have

$$
\begin{aligned}
\|V\|_{z-\bar{z}, \rho-\bar{\rho}} & =\sum_{\Lambda \in \tau}\left\|V_{\Lambda}\right\|_{\rho-\bar{\rho}} e^{(z-\bar{z})[\Lambda]} \\
& \leq \sum_{\Lambda \in \tau} \frac{\Gamma(\bar{\rho}) \Delta^{4}([\Lambda])}{\alpha}\left\|R_{\Lambda}\right\|_{\rho} e^{z[\Lambda]-\bar{z}[\Lambda]} \\
& \leq \frac{\Gamma(\bar{\rho}) \Gamma(\bar{z})}{\alpha}\|R\|_{z, \rho} .
\end{aligned}
$$


By Remark of Lemma 3.3, we have $\|B\| \cdot\left\|B^{-1}\right\| \leq c_{0}$, where $c_{0}>0$ is a constant. Then, by using Lemmas 3.1 and 3.2, we can write

$$
\|P\|_{z-\bar{z}, \rho-\bar{\rho}} \leq\|B\|\|V\|_{z-\bar{z}, \rho-\bar{\rho}}\left\|B^{-1}\right\|
$$

and

$$
\|R\|_{z, \rho} \leq\left\|B^{-1}\right\|\|Q\|_{z, \rho}\|B\|
$$

Thus,

$$
\|P\|_{z-\bar{z}, \rho-\bar{\rho}} \leq c \frac{\Gamma(\bar{z}) \Gamma(\bar{\rho})}{\alpha}\|Q\|_{z, \rho} .
$$

Now we prove that $P=\sum_{\Lambda \in \tau} P_{\Lambda}$ is Hamiltonian. To prove $P$ is Hamiltonian, we need to prove that $P_{J}$ is symmetric in $P_{J}=J^{-1} P$. As $J A$ and $Q=\sum_{\Lambda \in \tau} Q_{\Lambda}$ are Hamiltonian, then by definition, we can write $Q=J Q_{J}$, where $A$ and $Q_{J}$ are symmetric. Below we prove that $P_{J}$ is symmetric. Substituting $P=J P_{J}$ and $Q=J Q_{J}$ into equation (14), we have

$$
\dot{P}_{J}=A J P_{J}-P_{J} J A+Q_{J}
$$

and transposing equation (16), we have

$$
\dot{P}_{J}^{T}=A J P_{J}^{T}-P_{J}^{T} J A+Q_{J}
$$

It is easy to see that $J P_{J}$ and $J P_{J}^{T}$ are solutions of equation (14); moreover, $\overline{J P_{J}}=\overline{J P_{J}^{T}}=0$. Since the solution of equation (14) is unique with $\bar{P}=0$, we have that $J P_{J}=J P_{J}^{T}$, which proves that $P$ is Hamiltonian.

\section{Lemma 3.7 Consider the Hamiltonian system}

$$
\frac{d x}{d t}=J[A+\varepsilon Q(t)] x+\varepsilon g(t)+h(x, t),
$$

where $J A$ is a Hamiltonian matrix of dimension $2 N \times 2 N, J A \in B_{\varsigma}\left(A_{1}\right)$ with $\varsigma$ being given by Lemma 3.3, and $\lambda_{s}$ are eigenvalues of $J A$ with $\left|\lambda_{s}\right| \geq \eta_{3}>0, \forall 1 \leq s \leq 2 N$. Suppose that $Q(t)=\sum_{\Lambda \in \tau} Q_{\Lambda}(t), g(t)=\sum_{\Lambda \in \tau} g_{\Lambda}(t)$ are analytic almost-periodic on $D_{\rho}$, and $h(x, t)=$ $\sum_{\Lambda \in \tau} h_{\Lambda}(x, t)$ is an almost-periodic analytic matrix with respect to $t$ and $x$ on $\Delta_{b, \rho}$ with frequencies $\omega=\left(\omega_{1}, \omega_{2}, \ldots\right)$ and has a finite spatial structure $(\tau,[\cdot])$. Suppose also that $(x, t)$ is analytic with respect to $x$ on $B_{b}(0)$ and satisfies $\left\|D_{x x} h(x, t, \varepsilon)\right\| \leq K, \forall x \in B_{b}(0)$. Moreover,

$$
\left|\lambda_{s}-\sqrt{-1}\langle k, \omega\rangle\right| \geq \frac{\alpha}{\Delta^{4}(|k|) \Delta^{4}([k])}
$$

$\forall k \in \mathbf{Z}^{\mathbf{N}} \backslash\{0\}$, with a constant $\alpha>0$ and an approximation function $\Delta(t)$. Let $0<\bar{\rho}<\rho$, $0<\bar{z}<z$. Then, a symplectic change of variables $x=y+\underline{x}$ exists, so that the Hamiltonian system (17) can be transformed into the Hamiltonian system

$$
\frac{d y}{d t}=J\left[A+\varepsilon Q^{*}\right] y+\varepsilon^{2} g^{*}(t)+h^{*}(y, t)
$$


such that

$$
\left\|Q^{*}\right\|_{z-\bar{z}, \rho-\bar{\rho}} \leq\|Q\|_{z, \rho}+c K \frac{\Gamma(\bar{z}) \Gamma(\bar{\rho})}{\alpha}\|g\|_{z, \rho}
$$

and

$$
\left\|g^{*}\right\|_{z-\bar{z}, \rho-\bar{\rho}} \leq c \frac{\Gamma(\bar{z}) \Gamma(\bar{\rho})}{\alpha}\|Q\|_{z, \rho}\|g\|_{z, \rho}+c K\left(\frac{\Gamma(\bar{z}) \Gamma(\bar{\rho})}{\alpha}\right)^{2}\|g\|_{z, \rho}^{2}
$$

where $y \in B_{b_{1}}(0), b_{1}=b-\|\underline{x}\|_{z-\bar{z}, \rho-\bar{\rho}}$, and $\Gamma(\rho)=\sup _{t \geq 0}\left[\Delta^{4}(t) e^{-\rho t}\right.$.

Proof Consider that the equation $\frac{d x}{d t}=J A x+\varepsilon g(t)$ has solution $\underline{x}$. Using Lemma 3.4, we get

$$
\|\underline{x}\|_{z-\bar{z}, \rho-\bar{\rho}} \leq c \varepsilon \frac{\Gamma(\bar{z}) \Gamma(\bar{\rho})}{\alpha}\|g\|_{z, \rho} .
$$

Using the symplectic transformation $x=y+\underline{x}$, equation (17) becomes

$$
\frac{d y}{d t}=J\left[A+\varepsilon Q^{*}\right] y+\varepsilon^{2} g^{*}(t)+h^{*}(y, t)
$$

where

$$
\begin{aligned}
& g^{*}(t)=\frac{1}{\varepsilon^{2}} h(\underline{x}, t)+\frac{1}{\varepsilon} J Q(t) \underline{x}(t), \\
& Q^{*}(t)=Q(t)+\frac{1}{\varepsilon} D_{x} h(\underline{x}, t), \\
& h^{*}(y, t)=h(\underline{x}(t)+y, t)-h(\underline{x}, t)-J D_{x} h(\underline{x}(t), t) y .
\end{aligned}
$$

By Lemmas 3.4 and 3.5, we have

$$
\begin{aligned}
\left\|Q^{*}\right\|_{z-\bar{z}, \rho-\bar{\rho}} & \leq\|Q\|_{z, \rho}+\frac{1}{\varepsilon} K\|\underline{x}\|_{z-\bar{z}, \rho-\bar{\rho}} \\
& \leq\|Q\|_{z, \rho}+c K \frac{\Gamma(\bar{z}) \Gamma(\bar{\rho})}{\alpha}\|g\|_{z, \rho} .
\end{aligned}
$$

For the estimation of $\left\|g^{*}\right\|_{z-\bar{z}, \rho-\bar{\rho}}$, by Lemmas 3.4 and 3.5, we have

$$
\begin{aligned}
\left\|g^{*}\right\|_{z-\bar{z}, \rho-\bar{\rho}} & \leq c \frac{K}{2 \varepsilon^{2}}\left\|\underline{x}_{z-\bar{z}, \rho-\bar{\rho}}^{2}+\frac{1}{\varepsilon}\right\| Q \|_{z-\bar{z}, \rho-\bar{\rho}} \\
& \leq c \frac{\Gamma(\bar{z}) \Gamma(\bar{\rho})}{\alpha}\|Q\|_{z, \rho}\|g\|_{z, \rho}+c K\left(\frac{\Gamma(\bar{z}) \Gamma(\bar{\rho})}{\alpha}\right)^{2}\|g\|_{z, \rho}^{2} .
\end{aligned}
$$

Lemma 3.8 Let $\left\{\delta_{m}\right\}$ be a sequence of real positive numbers such that

$$
\delta_{m+1} \leq\left(\bar{\gamma} r^{m}\right)^{\bar{\gamma} r^{m}} \delta_{m}^{2}
$$

for all $m \geq 0$, where $\bar{\gamma}>0$ and $1<r<2$. Then

$$
\delta_{m} \leq\left[\left(\bar{\gamma} r^{\frac{r}{2-r}}\right)^{\frac{\bar{\gamma}}{2-r}} \delta_{0}\right]^{2 m}
$$

For the proof, see [5]. 
Lemma 3.9 Let $f:[-\varepsilon, \varepsilon] \rightarrow \mathbf{C}$ be Lipschitz from above (with constant $C_{f}$ ) and from below (with constant $c_{f}$ ), that is,

$$
|f(u)-f(v)| \leq C_{f}|u-v|, \quad|f(u)-f(v)| \geq c_{f}|u-v| .
$$

Let $g:[-\varepsilon, \varepsilon] \rightarrow \mathbf{C}$ be another Lipschitz from above (with constant $\delta<c_{f}$ ), that is,

$$
|g(u)-g(v)| \leq \delta|u-v|
$$

Then $h=f+g$ is a Lipschitz function from above (with constant $C_{f}+\delta$ ) and from below (with constant $c_{f}-\delta$ )

$$
|h(u)-h(v)| \leq\left(C_{f}+\delta\right)|u-v|, \quad|h(u)-h(v)| \geq\left(c_{f}-\delta\right)|u-v| .
$$

The proof is elementary.

Lemma 3.10 Suppose that $B_{1}$ has the eigenvalues $\lambda_{1}^{1}, \lambda_{2}^{1}, \ldots, \lambda_{2 N}^{1}$ which satisfy $\left|\lambda_{s}^{1}\right|>2 v$, $\left|\lambda_{s}^{1}-\lambda_{j}^{1}\right|>2 v$, and $v>0$ with $s \neq j$. Suppose that $A_{0}(\varepsilon)$ satisfies $\left\|A_{0}-B_{1}\right\|<\varsigma$ seen in Lemma 3.3 and $A_{0}(\varepsilon)$ relies on $\varepsilon$ with constant $L_{A_{0}}$ in a Lipschitz way. Suppose that $B(\varepsilon)$ is the transformation that diagonalizes $A_{0}(\varepsilon)$ (as in Lemma 3.3). Then there exist constants $C_{1}, C_{2}>0$ such that

$$
\begin{gathered}
\left\|B\left(\varepsilon_{1}\right)-B\left(\varepsilon_{2}\right)\right\| \leq C_{1} L_{A_{0}}\left|\varepsilon_{1}-\varepsilon_{2}\right|, \\
\left\|B\left(\varepsilon_{1}\right)-B\left(\varepsilon_{2}\right)\right\| \leq C_{1} L_{A_{0}}\left|\varepsilon_{1}-\varepsilon_{2}\right|, \\
\left\|\lambda_{j}\left(\varepsilon_{1}\right)-\lambda_{j}\left(\varepsilon_{2}\right)\right\| \leq C_{2} L_{A_{0}}\left|\varepsilon_{1}-\varepsilon_{2}\right|,
\end{gathered}
$$

where $\lambda_{j}(\varepsilon)$ for all $1 \leq j \leq 2 N$ denote the eigenvalues of $A_{0}(\varepsilon)$.

Lemma 3.11 Let $\left\{a_{m}\right\}_{m}$ be a sequence of positive real numbers which satisfy $\left.\left.a_{m} \in\right] 0,1\right]$, $\left.\left.\prod_{m=0}^{\infty} a_{m}=a \in\right] 0,1\right]$. Let $\left\{b_{m}\right\}_{m}$ be another sequence of positive real numbers satisfying $\prod_{m=0}^{\infty} b_{m}=b<+\infty$. Consider the new sequence $\left\{v_{m}\right\}_{m}$ defined by $v_{m+1}=a_{m} v_{m}-b_{m}$. Then the sequence $\left\{v_{m}\right\}_{m}$ approaches to a limit value $v_{\infty}$ which satisfies $v_{\infty} \geq a v_{0}-b$.

For the proof of Lemmas 3.10 and 3.11, see [5].

\section{The first KAM step}

Let $A_{0}=J A, Q_{0}(t)=J Q(t)$ be Hamiltonian matrices. First of all, for equation (1), the possible multiple eigenvalues of $A_{0}$ are changed into distinct eigenvalues and the coefficient $\varepsilon$ becomes $\varepsilon^{2}$ in $Q_{0}(t)$ and $g(t)$. In the following, to simplify notations, $c>0$ denotes the different constants. Then the Hamiltonian system (1) can be rewritten as follows:

$$
\frac{d x}{d t}=\left[A_{0}+\varepsilon Q_{0}(t)\right] x+\varepsilon g(t)+h(x, t)
$$

where $x \in B_{b}(0), Q_{0}$ and $g$ are analytic almost-periodic on $D_{\rho}$, and $h$ is analytic almostperiodic on $\Delta_{b, \rho}$ with spatial structure $(\tau,[\cdot])$. By using the symplectic transformation $x=$ 
$\underline{x}_{0}+y$, where $\underline{x}_{0}$ satisfies $\frac{d \underline{x}_{0}}{d t}=A_{0} \underline{x}_{0}+\varepsilon g(t)$ on $D_{\rho-\bar{\rho}}$, then system (18) becomes

$$
\frac{d y}{d t}=\left[A_{0}+\varepsilon Q^{*}(t)\right] y+\varepsilon^{2} g^{*}(t)+h^{*}(y, t)
$$

where

$$
\begin{aligned}
& Q^{*}=Q_{0}(t)+\frac{1}{\varepsilon} D_{x} h\left(\underline{x}_{0}, t\right), \\
& g^{*}(t)=\frac{1}{\varepsilon^{2}} h\left(\underline{x}_{0}, t\right)+\frac{1}{\varepsilon} Q_{0}(t) \underline{x}_{0}(t), \\
& h^{*}=h\left(\underline{x}_{0}+y, t\right)-h\left(\underline{x}_{0}, t\right)-J D_{x} h\left(\underline{x}_{0}, t\right) y .
\end{aligned}
$$

By using equation (8) and Lemma 3.4, we have

$$
\left\|\underline{x}_{0}\right\|_{z-\bar{z}, \rho-\bar{\rho}} \leq c \varepsilon \frac{\Gamma(\bar{z}) \Gamma(\bar{\rho})}{\alpha_{0}}\|g\|_{z, \rho} .
$$

By defining the average of $Q^{*}(t)$ as $\bar{Q}^{*}$, equation (19) can be rewritten as follows:

$$
\frac{d y}{d t}=\left[A_{1}+\varepsilon \widetilde{Q}(t)\right] y+\varepsilon^{2} \widetilde{g}(t)+\widetilde{h}(y, t)
$$

where $A_{1}=A_{0}+\varepsilon \bar{Q}^{*}, Q^{*}(t)=\widetilde{Q}(t)+\bar{Q}^{*}, \widetilde{g}=g^{*}$, and $\widetilde{h}=h^{*}$. By using the conditions of Theorem 2.1, $A_{1}$ has $2 N$ different eigenvalues $\lambda_{1}, \lambda_{2}, \ldots, \lambda_{2 N}$ which satisfy $\left|\lambda_{k_{1}}^{1}(\varepsilon)-\lambda_{k_{2}}^{1}(\varepsilon)\right| \geq$ $2 \eta \varepsilon>0, k_{1} \neq k_{2}$. Now, using the symplectic change of variables $y=e^{\varepsilon P_{0}(t)} x_{1}$, system (21) is transformed into the system

$$
\begin{aligned}
\frac{d x_{1}}{d t}= & {\left[e^{-\varepsilon P_{0}}\left(A_{1}+\varepsilon \widetilde{Q}-\varepsilon \dot{P}_{0}\right) e^{\varepsilon P_{0}}+e^{-\varepsilon P_{0}}\left(\varepsilon \dot{P}_{0} e^{\varepsilon P_{0}(t)}-\frac{d}{d t} e^{\varepsilon P_{0}(t)}\right)\right] x_{1} } \\
& +e^{-\varepsilon P_{0}} \varepsilon^{2} \widetilde{g}(t)+e^{-\varepsilon P_{0}} \widetilde{h}\left(e^{\varepsilon P_{0}(t)} x_{1}, t\right),
\end{aligned}
$$

where $x \in B_{b_{1}}(0)$. By series expansion, we can denote

$$
e^{\varepsilon P_{0}}=I+\varepsilon P_{0}+W, \quad e^{-\varepsilon P_{0}}=I-\varepsilon P_{0}+\widetilde{W}
$$

where

$$
\begin{aligned}
& W=\frac{\left(\varepsilon P_{0}\right)^{2}}{2 !}+\frac{\left(\varepsilon P_{0}\right)^{3}}{3 !}+\cdots, \\
& \widetilde{W}=\frac{\left(\varepsilon P_{0}\right)^{2}}{2 !}-\frac{\left(\varepsilon P_{0}\right)^{3}}{3 !}+\cdots .
\end{aligned}
$$

Then the Hamiltonian system (22) can be rewritten as follows:

$$
\begin{aligned}
\frac{d x_{1}}{d t}= & \left(A_{1}+\varepsilon \widetilde{Q}-\varepsilon \dot{P_{0}}+\varepsilon A_{1} P_{0}-\varepsilon P_{0} A_{1}+\varepsilon^{2} Q_{1}\right) x_{1} \\
& +e^{-\varepsilon P_{0}} \varepsilon^{2} \widetilde{g}(t)+e^{-\varepsilon P_{0}} \widetilde{h}\left(e^{\varepsilon P_{0}(t)} x_{1}, t\right),
\end{aligned}
$$


where

$$
\begin{aligned}
Q_{1}= & -P_{0}\left(\widetilde{Q}-\dot{P_{0}}\right)+\left(\widetilde{Q}-\dot{P_{0}}\right) P_{0}-P_{0}\left(A_{1}+\varepsilon \widetilde{Q}-\varepsilon \dot{P_{0}}\right) P_{0} \\
& +\left(I-\epsilon P_{0}\right)\left(A_{1}+\varepsilon \widetilde{Q}-\varepsilon \dot{P_{0}}\right) \frac{W}{\varepsilon^{2}}+\frac{\widetilde{W}}{\varepsilon^{2}}\left(A_{1}+\varepsilon \widetilde{Q}-\varepsilon \dot{P_{0}}\right) e^{\varepsilon P_{0}} \\
& \left.+\frac{1}{\varepsilon^{2}} e^{-\varepsilon P_{0}}\left(\varepsilon \dot{P_{0}} e^{\varepsilon P_{0}(t)}-\frac{d}{d t} e^{\varepsilon P_{0}(t)}\right)\right) .
\end{aligned}
$$

We would like to have that

$$
\widetilde{Q}-\dot{P}_{0}+A_{1} P_{0}-P_{0} A_{1}=0
$$

which is equivalent to

$$
\dot{P}_{0}=A_{1} P_{0}-P_{0} A_{1}+\widetilde{Q} .
$$

Since $A_{0}, Q^{*}(t)$, and $\bar{Q}^{*}$ are Hamiltonian matrices, therefore $A_{1}=A_{0}+\varepsilon \bar{Q}^{*}$ and $\widetilde{Q}(t)=$ $Q^{*}(t)-\bar{Q}^{*}$ are also Hamiltonian matrices. Using Lemma 3.6, if

$$
\left|\lambda_{s}^{1}-\lambda_{j}^{1}-\sqrt{-1}\langle k, \omega\rangle\right| \geq \frac{\alpha_{1}}{\Delta^{4}(|k|) \Delta^{4}([k])}, \quad s \neq j, 0 \leq s, j \leq 2 N
$$

for all $k \in \mathbf{Z}^{\mathbf{N}} \backslash\{0\}, \alpha_{1}=\frac{\alpha_{0}}{2}$, then for equation (24), a unique almost-periodic Hamiltonian matrix $P_{0}=\sum P_{0 \Lambda}$ exists with the same spatial structure $(\tau,[\cdot])$ and the same frequencies as $\widetilde{Q}(t)$ on a smaller domain $D_{\rho-\bar{\rho}}$, which satisfies $\bar{P}_{0}=0$ and

$$
\left\|P_{0}\right\|_{z-\bar{z}, \rho-\bar{\rho}} \leq c \frac{\Gamma(\bar{z}) \Gamma(\bar{\rho})}{\alpha_{0}}\left\|Q^{*}\right\|_{z, \rho} .
$$

Therefore, by equation (24), the Hamiltonian system (23) can be rewritten as follows:

$$
\frac{d x_{1}}{d t}=\left[A_{1}+\varepsilon^{2} Q_{1}(t)\right] x_{1}+\varepsilon^{2} g_{1}(t)+h_{1}\left(x_{1}, t\right)
$$

where $g_{1}(t)=e^{-\varepsilon P_{0}} \widetilde{g}(t), h_{1}\left(x_{1}, t\right)=e^{-\varepsilon P_{0}} \widetilde{h}\left(e^{\varepsilon P_{0}(t)} x_{1}, t\right)$, and

$$
\begin{aligned}
Q_{1}= & -P_{0}\left(P_{0} A_{1}-A_{1} P_{0}\right)+\left(P_{0} A_{1}-A_{1} P_{0}\right) P_{0}-P_{0}\left(A_{1}+\varepsilon\left(P_{0} A_{1}-A_{1} P_{0}\right)\right) P_{0} \\
& +\left(I-\varepsilon P_{0}\right)\left(A_{1}+\varepsilon\left(P_{0} A_{1}-A_{1} P_{0}\right)\right) \frac{W}{\varepsilon^{2}} \\
& +\frac{\widetilde{W}}{\varepsilon^{2}}\left(A_{1}+\varepsilon\left(P_{0} A_{1}-A_{1} P_{0}\right)\right) e^{\varepsilon P_{0}}+\frac{1}{\varepsilon^{2}} e^{-\varepsilon P_{0}}\left(\varepsilon \dot{P_{0}} e^{\varepsilon P_{0}}-\frac{d\left(e^{\varepsilon P_{0}}\right)}{d t}\right) .
\end{aligned}
$$

Hence, the symplectic transformation is $T_{0} x_{1}=\underline{x}_{0}+e^{\varepsilon P_{0}} x_{1}=\varphi_{0}(t, \varepsilon)+\psi_{0}(t, \varepsilon) x_{1}$. If $\left\|\varepsilon P_{0}\right\|_{z-\bar{z}, \rho-\bar{\rho}} \leq \frac{1}{2}$, then, by equations (20) and (26), we have

$$
\begin{aligned}
& \left\|\varphi_{0}\right\|_{z-\bar{z}, \rho-\bar{\rho}} \leq c \varepsilon \frac{\Gamma(\bar{z}) \Gamma(\bar{\rho})}{\alpha_{0}}\|g\|_{z, \rho}, \\
& \left\|\psi_{0}-I\right\|_{z-\bar{z}, \rho-\bar{\rho}} \leq c \varepsilon \frac{\Gamma(\bar{z}) \Gamma(\bar{\rho})}{\alpha_{0}}\left\|Q^{*}\right\| \|_{z, \rho} .
\end{aligned}
$$


Thus, under the symplectic change of variables $x=T_{0} x_{1}$, the Hamiltonian system (18) becomes Hamiltonian system (27). This completes the first KAM step.

\section{Proof of the main result}

\subsection{The proof of Theorem 2.1}

Now we will consider the standard iteration step, the proof of which is almost similar to the first KAM step. In the first step, we proved that $A_{1}$ has $2 N$ different eigenvalues and $\varepsilon^{2} Q_{1}(t)$ and $\varepsilon^{2} g_{1}(t)$ are smaller perturbations. Now the KAM method will be used to prove Theorem 2.1 and we will use a similar process as that in [5] and [8]. For simplification of notations, here $c>0$ denotes the different constants. For $m$ th step, consider the Hamiltonian system

$$
\frac{d x_{m}}{d t}=\left[A_{m}+\varepsilon^{2^{m}} Q_{m}(t, \varepsilon)\right] x_{m}+\varepsilon^{2^{m}} g_{m}(t, \varepsilon)+h_{m}\left(x_{m}, t, \varepsilon\right),
$$

where $m \geq 1, x_{m} \in B_{b_{m}}(0), Q_{m}$ and $g_{m}$ are analytic almost-periodic on $D_{\rho_{m}}$, and $h_{m}$ is analytic almost-periodic on $\Delta_{b_{m}, \rho_{m}}$ with basic frequencies $\omega=\left(\omega_{1}, \omega_{2}, \ldots\right)$ and has the spatial structure ( $\tau,[\cdot]), A_{m} \in B_{\zeta}\left(A_{1}\right)$, and $\lambda_{s}^{m}$ are eigenvalues of $A_{m}$ with $\left|\lambda_{s}^{m}(\varepsilon)-\lambda_{j}^{m}(\varepsilon)\right| \geq \eta \varepsilon>0$, $\forall s \neq j$ and $\left|\lambda_{s}^{m}(\varepsilon)\right| \geq \eta_{3}>0$. Using the change of variables $x_{m}=\underline{x}_{m}+y$, where $\underline{x}_{m}$ satisfies $\frac{d \underline{x}_{m}}{d t}=A_{m} \underline{x}_{m}+\varepsilon^{2^{m}} g_{m}(t)$ on $D_{\rho_{m}-\bar{\rho}_{m}}$, the Hamiltonian system (30) can be written as follows:

$$
\frac{d y}{d t}=\left[A_{m}+\varepsilon^{2^{m}} Q_{m}^{*}(t)\right] y+\varepsilon^{2^{m+1}} g_{m}^{*}(t)+h_{m}^{*}(y, t)
$$

where

$$
\begin{aligned}
& Q_{m}^{*}(t)=Q_{m}(t)+\frac{1}{\varepsilon^{2^{m}}} D_{x} h_{m}\left(\underline{x}_{m}, t\right), \\
& g_{m}^{*}(t)=\frac{1}{\varepsilon^{2^{m+1}}} h_{m}\left(\underline{x}_{m}, t\right)+\frac{1}{\varepsilon^{2^{m}}} Q_{m}(t) \underline{x}_{m}(t), \\
& h_{m}^{*}(y, t)=h_{m}\left(\underline{x}_{m}+y, t\right)-h_{m}\left(\underline{x}_{m}, t\right)-D_{x} h_{m}\left(\underline{x}_{m}, t\right) y .
\end{aligned}
$$

By Lemma 3.4, if

$$
\left|\lambda_{s}^{m}-\sqrt{-1}\langle k, \omega\rangle\right| \geq \frac{\alpha_{m}}{\Delta^{4}(|k|) \Delta^{4}([k])}
$$

$\forall k \in \mathbf{Z}^{\mathbf{N}} \backslash\{0\}, \alpha_{m}=\frac{\alpha_{0}}{2^{m}}$, and $\Delta$ is an approximation function, then we have

$$
\left\|\underline{x}_{m}\right\|\left\|_{z_{m}-\bar{z}_{m}, \rho_{m}-\bar{\rho}_{m}} \leq c \varepsilon^{2^{m}} \frac{\Gamma\left(\bar{z}_{m}\right) \Gamma\left(\bar{\rho}_{m}\right)}{\alpha_{m}}\right\| g_{m} \|_{z_{m}, \rho_{m}},
$$

where $0<\bar{z}_{m}<z_{m}, 0<\bar{\rho}_{m}<\rho_{m}$, and $c>0$ is a constant. By defining the average of $Q_{m}^{*}(t)$ as $\bar{Q}_{m}^{*}$, equation (31) can be rewritten as follows:

$$
\frac{d y}{d t}=\left[A_{m+1}+\varepsilon^{2^{m}} \widetilde{Q}_{m}(t)\right] y+\varepsilon^{2^{m+1}} \widetilde{g}_{m}(t)+\widetilde{h}_{m}(y, t),
$$

where $A_{m+1}=A_{m}+\varepsilon \bar{Q}_{m}^{*}, Q_{m}^{*}(t)=\widetilde{Q}_{m}(t)+\bar{Q}_{m}^{*}, \widetilde{g}_{m}=g_{m}^{*}$, and $\widetilde{h}_{m}=h_{m}^{*}$. 
Now consider $\lambda_{1}^{m+1}, \ldots, \lambda_{2 N}^{m+1}$ to be the different eigenvalues of $A_{m+1}$ which satisfy $\mid \lambda_{k_{1}}^{m+1}$ $\lambda_{k_{2}}^{m+1} \mid \geq 2 \eta \varepsilon>0, k_{1} \neq k_{2}$.

By applying the symplectic transformation $y=e^{\varepsilon^{2^{m}} P_{m}(t)} x_{m+1}$, system (33) is changed into

$$
\begin{aligned}
\frac{d x_{m+1}}{d t}= & {\left[e^{-\varepsilon^{2^{m}} P_{m}}\left(A_{m+1}+\varepsilon^{2^{m}} \widetilde{Q}_{m}-\varepsilon^{2^{m}} \dot{P_{m}}\right) e^{\varepsilon^{2^{m}} P_{m}}\right.} \\
& \left.+e^{-\varepsilon^{2^{m}} P_{m}}\left(\varepsilon^{2^{m}} \dot{P_{m}} e^{\varepsilon^{\varepsilon^{m}} P_{m}(t)}-\frac{d}{d t} e^{\varepsilon^{\varepsilon^{m}} P_{m}(t)}\right)\right] x_{m+1} \\
& +e^{-\varepsilon^{2^{m}} P_{m}} \varepsilon^{2^{m+1}} \widetilde{g}_{m}(t)+e^{-\varepsilon^{2^{m}} P_{m}} \widetilde{h}_{m}\left(e^{\varepsilon^{2^{m}} P_{m}(t)} x_{m+1}, t\right),
\end{aligned}
$$

where $x_{m+1} \in B_{b_{m+1}}(0)$. By series expansion, we can denote

$$
e^{\varepsilon^{2^{m}} P_{m}}=I+\varepsilon^{2^{m}} P_{m}+W_{m}, \quad e^{-\varepsilon^{2^{m}} P_{m}}=I-\varepsilon^{2^{m}} P_{m}+\widetilde{W}_{m},
$$

where

$$
W_{m}=\frac{\left(\varepsilon^{2^{m}} P_{m}\right)^{2}}{2 !}+\frac{\left(\varepsilon^{2^{m}} P_{m}\right)^{3}}{3 !}+\cdots, \quad \widetilde{W}_{m}=\frac{\left(\varepsilon^{2^{m}} P_{m}\right)^{2}}{2 !}-\frac{\left(\varepsilon^{2^{m}} P_{m}\right)^{3}}{3 !}+\cdots
$$

Then system (34) can be rewritten as follows:

$$
\begin{aligned}
\frac{d x_{m+1}}{d t}= & {\left[A_{m+1}+\varepsilon^{2^{m}} \widetilde{Q}_{m}-\varepsilon^{2^{m}} \dot{P}_{m}+\varepsilon^{2^{m}} A_{m+1} P_{m}-\varepsilon^{2^{m}} P_{m} A_{m+1}+\varepsilon^{2^{m+1}} Q_{m+1}(t)\right] x_{m+1} } \\
& +e^{-\varepsilon^{2^{m}} P_{m}} \varepsilon^{2^{m+1}} \widetilde{g}_{m}(t)+e^{-\varepsilon^{2^{m}} P_{m}} \widetilde{h}_{m}\left(e^{\varepsilon^{2^{m}} P_{m}(t)} x_{m+1}, t\right),
\end{aligned}
$$

where

$$
\begin{aligned}
Q_{m+1}(t)= & -P_{m}\left(\widetilde{Q}_{m}-\dot{P}_{m}\right)+\left(\widetilde{Q}_{m}-\dot{P}_{m}\right) P_{m}-P_{m}\left(A_{m+1}+\varepsilon^{2^{m}}\left(\widetilde{Q}_{m}-\dot{P}_{m}\right)\right) P_{m} \\
& +\left(I-\epsilon^{2^{m}} P_{m}\right)\left(A_{m+1}+\varepsilon^{2^{m}}\left(\widetilde{Q}_{m}-\dot{P}_{m}\right)\right) \frac{W_{m}}{\varepsilon^{2^{m+1}}} \\
& +\frac{\widetilde{W}_{m}}{\varepsilon^{2^{m+1}}}\left(A_{m+1}+\varepsilon^{2^{m}}\left(\widetilde{Q}_{m}-\dot{P}_{m}\right)\right) e^{\varepsilon^{2^{m}} P_{m}} \\
& +\frac{1}{\varepsilon^{2^{m+1}}} e^{-\varepsilon^{2^{m}} P_{m}}\left(\varepsilon^{2^{m}} \dot{P}_{m} e^{\varepsilon^{\varepsilon^{m}} P_{m}}-\frac{d}{d t} e^{\varepsilon^{2^{m}} P_{m}}\right) .
\end{aligned}
$$

We would like to have

$$
\widetilde{Q}_{m}-\dot{P}_{m}+A_{m+1} P_{m}-P_{m} A_{m+1}=0 .
$$

This can be rewritten as

$$
\dot{P}_{m}=A_{m+1} P_{m}-P_{m} A_{m+1}+\widetilde{Q}_{m}
$$

Since $A_{m}, Q_{m}^{*}(t)$, and $\bar{Q}_{m}^{*}$ are Hamiltonian, therefore $A_{m+1}$ and $\widetilde{Q}_{m}(t)$ are Hamiltonian.

By Lemma 3.6, if

$$
\left|\lambda_{s}^{m+1}-\lambda_{j}^{m+1}-\sqrt{-1}\langle k, \omega\rangle\right| \geq \frac{\alpha_{m}}{\Delta^{4}(|k|) \Delta^{4}([k])}, \quad k \in \mathbf{Z}^{\mathbf{N}} \backslash\{0\}
$$


and for different eigenvalues $\lambda_{1}^{m+1}, \ldots, \lambda_{2 N}^{m+1}$ of $A_{m+1}$ with $\left|\lambda_{s}^{m+1}-\lambda_{j}^{m+1}\right| \geq \eta \varepsilon, s \neq j 0 \leq$ $s, j \leq 2 N$, then for equation (36), there exists a unique almost-periodic matrix $P_{m}(t)=$ $\sum_{\Lambda \in \tau} P_{m \Lambda}(t)$ on $D_{\rho_{m}-\bar{\rho}_{m}}$ with frequencies $\omega$ and spatial structure $(\tau,[\cdot])$, which satisfies

$$
\left\|P_{m}\right\|_{z_{m}-\overline{z_{m}}, \rho_{m}-\bar{\rho}_{m}} \leq c \frac{\Gamma\left(\bar{z}_{m}\right) \Gamma\left(\bar{\rho}_{m}\right)}{\alpha_{m}}\left\|Q_{m}^{*}\right\| \|_{z_{m}, \rho_{m}} .
$$

Then the Hamiltonian system (35) becomes

$$
\frac{d x_{m+1}}{d t}=\left[A_{m+1}+\varepsilon^{2^{m+1}} Q_{m+1}(t)\right] x_{m+1}+\varepsilon^{2^{m+1}} g_{m+1}(t)+h_{m+1}\left(x_{m+1}, t\right)
$$

where $g_{m+1}(t)=e^{-\varepsilon^{2^{m}} P_{m}} \widetilde{g}_{m}(t), h_{m+1}\left(x_{m+1}, t\right)=e^{-\varepsilon^{2^{m}} P_{m}} \widetilde{h}_{m}\left(e^{\varepsilon^{2^{m}} P_{m}(t)} x_{m+1}, t\right)$, and by using $\widetilde{Q}_{m}-$ $\dot{P}_{m}=P_{m} A_{m+1}-A_{m+1} P_{m}$, we have

$$
\begin{aligned}
Q_{m+1}(t)= & -P_{m}\left(P_{m} A_{m+1}-A_{m+1} P_{m}\right)+\left(P_{m} A_{m+1}-A_{m+1} P_{m}\right) P_{m} \\
& -P_{m}\left(A_{m+1}+\varepsilon^{2^{m}}\left(P_{m} A_{m+1}-A_{m+1} P_{m}\right)\right) P_{m} \\
& +\left(I-\epsilon^{2^{m}} P_{m}\right)\left(A_{m+1}+\varepsilon^{2^{m}}\left(P_{m} A_{m+1}-A_{m+1} P_{m}\right)\right) \frac{W_{m}}{\varepsilon^{2^{m+1}}} \\
& +\frac{\widetilde{W}_{m}}{\varepsilon^{2^{m+1}}}\left(A_{m+1}+\varepsilon^{2^{m}}\left(P_{m} A_{m+1}-A_{m+1} P_{m}\right)\right) e^{\varepsilon^{2^{m}} P_{m}} \\
& +\frac{1}{\varepsilon^{2^{m+1}}} e^{-\varepsilon^{2^{m}} P_{m}}\left(\varepsilon^{2^{m}} P_{m} e^{\varepsilon^{\varepsilon^{m}} P_{m}}-\frac{d}{d t} e^{\varepsilon^{2^{m}} P_{m}(t)}\right) .
\end{aligned}
$$

Thus, the symplectic transformation is $T_{m} x_{m+1}=\underline{x}_{m}+e^{\varepsilon^{2^{m}} P_{m}} x_{m+1}=\varphi_{m}(t)+\psi_{m}(t) x_{m+1}$. If $\left\|\varepsilon^{2^{m}} P_{m}\right\| \|_{z_{m}-\bar{z}_{m}, \rho_{m}-\bar{\rho}_{m}} \leq \frac{1}{2}$, then by equations (32) and (37), we have

$$
\begin{aligned}
& \left\|\varphi_{m}\right\|_{z_{m}-\bar{z}_{m}, \rho_{m}-\bar{\rho}_{m}} \leq c \varepsilon^{2^{m}} \frac{\Gamma\left(\bar{z}_{m}\right) \Gamma\left(\bar{\rho}_{m}\right)}{\alpha_{m}}\left\|g_{m}\right\|_{z_{m}, \rho_{m}}, \\
& \left\|\psi_{m}-I\right\|_{z_{m}-\bar{z}_{m}, \rho_{m}-\bar{\rho}_{m}} \leq c \varepsilon^{2^{m}} \frac{\Gamma\left(\bar{z}_{m}\right) \Gamma\left(\bar{\rho}_{m}\right)}{\alpha_{m}}\left\|Q_{m}^{*}\right\| \|_{z_{m}, \rho_{m}} .
\end{aligned}
$$

So, using the symplectic change of variables $x_{m}=T_{m} x_{m+1}$, system (30) is transformed into system (38).

\subsection{Iteration}

Now we estimate the bounds of $\left\|g_{m+1}\right\|_{m+1}$ and $\left\|Q_{m+1}\right\|_{m+1}$ as $m \rightarrow \infty$. For the $m$ th step, we choose

$$
\alpha_{m}=\frac{\alpha_{0}}{2^{m}}, \quad b_{m+1}=\frac{b_{m}-\left\|\underline{x}_{m}\right\|_{m}}{e^{\varepsilon^{2^{m}}\left\|P_{m}\right\|_{m+1}}}, \quad\|\| \cdot\left\|_{m}=\right\| \cdot\|\|_{z_{m}, \rho_{m}} .
$$

Also, suppose that $\bar{z}_{v} \downarrow 0$ and $\bar{\rho}_{\nu} \downarrow 0$ satisfy $\sum_{\nu=0}^{\infty} \bar{z}_{v}=\frac{1}{2} z$ and $\sum_{\nu=0}^{\infty} \bar{\rho}_{\nu}=\frac{1}{2} \rho$. And set $z_{m}=z-\sum_{v=1}^{m} \bar{z}_{v}, \rho_{m}=\rho-\sum_{v=1}^{m} \bar{\rho}_{v}$. Assume that

$$
\varphi(\rho)=\inf _{\rho_{1}+\rho_{2}+\cdots<\rho} \prod_{\nu=1}^{\infty}\left[\Gamma\left(\rho_{\nu}\right)\right]^{2^{-v-1}},
$$


then

$$
\varphi\left(\frac{1}{2} z\right)=\prod_{\nu=1}^{\infty}\left[\Gamma\left(\bar{z}_{\nu}\right)\right]^{2^{-\nu-1}}
$$

and

$$
\varphi\left(\frac{1}{2} \rho\right)=\prod_{\nu=1}^{\infty}\left[\Gamma\left(\bar{\rho}_{\nu}\right)\right]^{2^{-\nu-1}} .
$$

If $\left\|\varepsilon^{2^{m}} P_{m}\right\|_{m+1} \leq \frac{1}{2}$, we have

$$
b_{m+1} \geq \frac{b_{m}-\left\|\underline{x}_{m}\right\|_{m}}{1+2 \varepsilon^{2^{2}}\left\|P_{m}\right\|_{m+1}} .
$$

By using Lemma 3.11, equation (42), and for sufficiently small $\varepsilon$, we obtain $b_{\infty}=$ $\lim _{m \rightarrow \infty} b_{m} \geq \sigma$ with constant $\sigma>0$. By Lemma 3.7, we obtain

$$
\left\|Q_{m}^{*}\right\|_{m+1} \leq\left\|Q_{m}\right\|_{m}+c K_{m} \frac{\Gamma\left(\bar{z}_{m+1}\right) \Gamma\left(\bar{\rho}_{m+1}\right)}{\alpha_{m}}\left\|g_{m}\right\|_{m} .
$$

Therefore, by equations (37) and (43), we have

$$
\left\|P_{m}\right\|_{m+1} \leq c K_{m} \frac{\left(\Gamma\left(\bar{z}_{m+1}\right) \Gamma\left(\bar{\rho}_{m+1}\right)\right)^{2}}{\alpha_{m} \alpha_{m+1}}\left(\left\|Q_{m}\right\|_{m}+\left\|g_{m}\right\|_{m}\right) .
$$

Set

$$
\begin{aligned}
& c_{1}=\max \left\{c, \frac{8 c}{\alpha}\right\}, \quad c_{m}=\left[(m+1)^{2^{-(m+1)}} m^{2^{-m}} \cdots 2^{2^{-2}} \cdot 1^{2^{-1}}\right]^{2} \\
& \Phi_{m}(z)=\prod_{\nu=1}^{m+1}\left[\Gamma\left(\bar{z}_{\nu}\right)\right]^{2^{-\nu}}, \quad \Phi_{m}(\rho)=\prod_{\nu=1}^{m+1}\left[\Gamma\left(\bar{\rho}_{\nu}\right)\right]^{2^{-\nu}} .
\end{aligned}
$$

From [9], $c_{m}, \Phi_{m}(z), \Phi_{m}(\rho)$ are all convergent when $m$ goes to infinity. Consider

$$
\begin{aligned}
& \mathcal{M}_{1}=\max \left\{1, \sup _{m}\left(c_{1} c_{m} \Phi_{m}(z) \Phi_{m}(\rho)\right)\right\}\|Q(t)\|_{z, \rho}, \\
& \mathcal{M}_{2}=\max \left\{1, \sup _{m}\left(c_{1} c_{m} \Phi_{m}(z) \Phi_{m}(\rho)\right)\right\}\|g(t)\| \|_{z, \rho},
\end{aligned}
$$

and set

$$
\mathcal{M}=\max \left\{\mathcal{M}_{1}, \mathcal{M}_{2}\right\}
$$

Firstly, we calculate $\left\|g_{m+1}\right\|_{m+1}$. By Lemma 3.7, we have

$$
\begin{aligned}
& \left\|g_{m+1}\right\|_{m+1} \\
& \quad \leq c \frac{\Gamma\left(\bar{z}_{m+1}\right) \Gamma\left(\bar{\rho}_{m+1}\right)}{\alpha_{m}}\left(\left\|Q_{m}\right\|_{m}+c K_{m} \frac{\Gamma\left(\bar{z}_{m+1}\right) \Gamma\left(\bar{\rho}_{m+1}\right)}{\alpha_{m}}\left\|g_{m}\right\|_{m}\right)\left\|g_{m}\right\|_{m} .
\end{aligned}
$$

Now we estimate $\left\|Q_{m+1}\right\|_{m+1}$. 
If $\left\|\varepsilon^{2^{m}} P_{m}\right\|_{m+1} \leq \frac{1}{2}$, we have

$$
\left\|e^{ \pm \varepsilon^{\varepsilon^{m}} P_{m}}\right\|_{m+1} \leq 1+\left\|\varepsilon^{2^{m}} P_{m}\right\| \mid+\frac{\left\|\varepsilon^{2^{m}} P_{m}\right\|^{2}}{2 !}+\cdots \leq 2 .
$$

Moreover, we have that

$$
\begin{aligned}
& \varepsilon^{2^{m}} \dot{P_{m}} e^{\varepsilon^{\varepsilon^{m}} P_{m}}-\frac{d}{d t} e^{\varepsilon^{2^{m}} P_{m}(t)} \\
& =\left(\varepsilon^{2^{m}} \dot{P_{m}} e^{\varepsilon^{m} P_{m}}+\varepsilon^{2^{m}} \dot{P_{m}} \frac{\left(\varepsilon^{2^{m}} P_{m}\right)^{2}}{2 !}+\varepsilon^{2^{m}} \dot{P_{m}} \frac{\left(\varepsilon^{2^{m}} P_{m}\right)^{3}}{3 !}+\cdots\right) \\
& \quad-\frac{d\left(\frac{\left(\varepsilon^{2^{m}} P_{m}\right)^{2}}{2 !}+\frac{\left(\varepsilon^{m} P_{m}\right)^{3}}{3 !}+\cdots\right)}{d t} .
\end{aligned}
$$

If $\left\|\varepsilon^{\varepsilon^{m}} P_{m}\right\|_{m+1} \leq \frac{1}{2}$, by

$$
\left\|\frac{d}{d t}\left(\varepsilon^{2^{m}} P_{m}\right)^{n}\right\| \leq n\left\|\mid \varepsilon^{2^{m}} \dot{P_{m}}\right\|\|\| \varepsilon^{2^{m}} P_{m} \|^{n-1}, \quad \text { for } n \in \mathbf{Z}^{+},
$$

we obtain that

$$
\left\|\varepsilon^{2^{m}} \dot{P_{m}} \varepsilon^{\varepsilon^{2^{m}} P_{m}}-\frac{d}{d t} e^{\varepsilon^{2^{m}} P_{m}(t)}\left|\left\|_{m+1} \leq 4\right\|\right|\right\| \varepsilon^{2^{m}} \dot{P_{m}}\|\|_{m+1}\left\|\mid \varepsilon^{2^{m} P_{m}}\right\|_{m+1} .
$$

By equations (36) and (43), we have

$$
\begin{aligned}
& \left\|\varepsilon^{2^{m}} \dot{P_{m}} e^{\varepsilon^{2^{m}} P_{m}}-\frac{d}{d t} e^{\varepsilon^{2^{m}} P_{m}(t)}\right\| \|_{m+1} \\
& \leq c \varepsilon^{2^{m+1}}\left(\left\|P_{m}\right\|_{m+1}^{2}+\left\|P_{m}\right\|_{m+1}\left\|Q_{m}\right\|_{m}\right. \\
& \left.+\left\|P_{m}\right\|_{m+1} c K_{m} \frac{\Gamma\left(\bar{z}_{m+1}\right) \Gamma\left(\bar{\rho}_{m+1}\right)}{\alpha_{m}}\left\|g_{m}\right\|_{m}\right) .
\end{aligned}
$$

Also, if $\left\|\varepsilon^{2^{m}} P_{m}\right\|_{m+1} \leq \frac{1}{2}$, we have

$$
\left\|W_{m}\right\|_{m+1},\left\|\widetilde{W}_{m}\right\|_{m+1} \leq 2\left\|\mid \varepsilon^{2^{m}} P_{m}\right\|_{m+1}^{2} .
$$

So, by equations (40),(46), and (47), we have

$$
\begin{aligned}
& \left\|Q_{m+1}\right\|_{m+1} \\
& \quad \leq c K_{m} \frac{\Gamma\left(\bar{z}_{m+1}\right) \Gamma\left(\bar{\rho}_{m+1}\right)}{\alpha_{m}}\left(\left\|P_{m}\right\|_{m+1}^{2}+\left\|P_{m}\right\|_{m+1}\left\|Q_{m}\right\|_{m}+\left\|P_{m}\right\|\left\|_{m+1}\right\| g_{m} \|_{m}\right) .
\end{aligned}
$$

Then, by using equation (43), the above equation can be written as follows:

$$
\left\|Q_{m+1}\right\|_{m+1} \leq c K_{m}^{3} \frac{\left(\Gamma\left(\bar{z}_{m+1}\right) \Gamma\left(\bar{\rho}_{m+1}\right)\right)^{5}}{\alpha_{m}^{3} \alpha_{m+1}^{2}}\left(\left\|Q_{m}\right\|_{m}^{2}+\left\|g_{m}\right\|_{m}^{2}+\left\|Q_{m}\right\|_{m}\left\|g_{m}\right\|_{m}\right) .
$$


For the estimate of $\left\|Q_{m}\right\|_{m}$ and $\left\|g_{m}\right\|_{m}$, we define

$$
\delta_{m}=\max \left\{\left\|Q_{m}\right\|_{m},\left\|g_{m}\right\|_{m}\right\} .
$$

By, equations (45) and (48), we have that

$$
\delta_{m+1} \leq c K_{m}^{3} \frac{\left(\Gamma\left(\bar{z}_{m+1}\right) \Gamma\left(\bar{\rho}_{m+1}\right)\right)^{5}}{\alpha_{m}^{3} \alpha_{m+1}^{2}} \delta_{m}^{2}
$$

here $c>0$ is a constant depending on $\rho, z$, and $\mathcal{M}$.

If $\left\|\varepsilon^{2^{l}} P_{l}\right\| \leq \frac{1}{4}$ for $l=0,1,2, \ldots, m-1$, then we have $K_{m} \leq\left(\frac{9}{2}\right)^{m} K$ (as given below). It is immediate to check that we can find $\bar{\gamma}>0$ such that $\frac{c K_{m}^{3}}{\alpha_{m}^{3} \alpha_{m+1}^{2}}\left(\Gamma\left(\bar{z}_{m+1}\right) \Gamma\left(\bar{\rho}_{m+1}\right)\right)^{5} \leq\left(\bar{\gamma} r^{m}\right)^{\bar{\gamma} r^{m}}$, where $1<r<2$.

Using Lemma 3.8, we have $\delta_{m} \leq M^{2^{m}}$, where $M=\left(\bar{\gamma} r^{\frac{r}{2-r}}\right)^{\frac{\bar{\gamma}}{2-r}} \delta_{0}$. Thus, we have

$$
\left\|Q_{m}\right\|_{m} \leq M^{2^{m}}, \quad\|\| g_{m} \|_{m} \leq M^{2^{m}} .
$$

If $0<\varepsilon M<1$, then we obtain

$$
\lim _{m \rightarrow \infty} \varepsilon^{2^{m}}\left\|Q_{m}\right\|_{m}=0, \quad \lim _{m \rightarrow \infty} \varepsilon^{2^{m}}\left\|g_{m}\right\|_{m}=0 .
$$

Now, we bound $\left\|A_{m}\right\|$. By (43), we have

$$
\left\|A_{m+1}-A_{m}\right\| \leq\left\|\varepsilon^{2^{m}} Q_{m}^{*}\right\|_{m+1} \leq \varepsilon^{2^{m}}\left(\left\|Q_{m}\right\|_{m}+c K_{m} \frac{\Gamma\left(\bar{z}_{m+1}\right) \Gamma\left(\bar{\rho}_{m+1}\right)}{\alpha_{m}}\left\|g_{m}\right\|_{m}\right) .
$$

By $c K_{m} \frac{\Gamma\left(\bar{z}_{m+1}\right) \Gamma\left(\bar{\rho}_{m+1}\right)}{\alpha_{m}} \leq M_{1}^{2^{m}}$ with suitable constant $M_{1}>0$ and (50), if $\varepsilon M_{1} M<1$, then as $m \rightarrow \infty,\left\|A_{m+1}-A_{m}\right\| \rightarrow 0$. Hence, $A_{m}$ is convergent, as $m \rightarrow \infty$. Suppose

$$
\lim _{m \rightarrow \infty} A_{m}=A_{*} .
$$

Also, by (40), (41), (43), and (50), we have

$$
\lim _{m \rightarrow \infty}\left\|\varphi_{m}\right\|_{m+1}=0, \quad \lim _{m \rightarrow \infty}\left\|\psi_{m}-I\right\|_{m+1}=0 .
$$

Let $D_{\frac{1}{2} z, \Delta_{b, \frac{1}{2} \rho}}=\bigcap_{m=0}^{\infty} D_{z_{m}, \Delta_{b_{m}, \rho_{m}}}$. By equations (28), (29), (40), and (41), and by the conditions of Theorem 2.1, suppose $T^{m}=T_{0} \circ T_{1} \circ \cdots \circ T_{m-1}$. And the convergence of $T^{m}$ is easy to prove on $D_{\frac{1}{2} z, \Delta_{b, \frac{1}{2} \rho}}$ as $m \rightarrow \infty$. Let $T^{m} \rightarrow T$ on $D_{\frac{1}{2} z, \Delta_{b, \frac{1}{2} \rho}}$ as $m \rightarrow \infty$. To bound $\left\|P_{m}\right\|_{m+1}$. For (44), it is not difficult to choose $c K_{m} \frac{\left(\Gamma\left(\bar{z}_{m+1}\right) \Gamma\left(\overline{(}_{m+1}\right)\right)^{2}}{\alpha_{m} \alpha_{m+1}} \leq M_{2}^{2^{m}}$ with suitable constant $M_{2}>0$. So, by (50), if $\varepsilon M_{2} M<1$, we have

$$
\lim _{m \rightarrow \infty} \varepsilon^{2^{m}}\left\|P_{m}\right\|_{m+1}=0
$$

This allows us to have the condition $\varepsilon^{2^{m}}\left\|P_{m}\right\|_{m+1} \leq \frac{1}{4}$ without reducing the value of $\varepsilon$ at each step. Now we will show that $K_{m}$ is convergent for $m \rightarrow \infty$. By (38), we have

$$
\left\|D_{x_{m+1} x_{m+1}} h_{m+1}\right\|_{z_{m+1}, \Delta_{b_{m+1}, \rho_{m+1}}} \leq \frac{\left(1+2\left\|\varepsilon^{2^{m}} P_{m}\right\|_{m+1}\right)^{2}}{1-2\left\|\varepsilon^{2^{m}} P_{m}\right\|_{m+1}} K_{m} .
$$


So, if $\varepsilon^{2^{m}}\left\|P_{m}\right\|_{m+1} \leq \frac{1}{4}$, we already have $K_{m} \leq\left(\frac{9}{2}\right)^{m} K$. By the inequality $\frac{1}{1-x} \leq 1+2 x$, if $\frac{1}{4} \leq x \leq \frac{1}{2}$ and (55), we obtain

$$
K_{m+1} \leq\left(1+4 \varepsilon^{2^{m}}\left\|P_{m}\right\|_{m+1}\right)^{3} K_{m}
$$

Using the convergent bound of $\left\|P_{m}\right\|_{m+1}$ and since $K_{m} \leq\left(\frac{9}{2}\right)^{m} K$, then by (56) it is easy to obtain that the value of $K_{m}$ is convergent for $m \rightarrow \infty$. Suppose

$$
\lim _{m \rightarrow \infty} K_{m}=K_{*}
$$

Thus, $\lim _{m \rightarrow \infty} h_{m}=h_{*}(y, t)=O\left(y^{2}\right)$ as $y \rightarrow 0$.

Hence, using the symplectic change of variables $x=T y=\varphi(t)+\psi(t) y$ on $D_{\frac{1}{2} z, \Delta}, \frac{1}{2} \rho$, system (1) is changed into the Hamiltonian system (10).

\subsection{Measure estimate}

Now, for small enough $\varepsilon_{0}$, we will prove that the non-resonant conditions

$$
\left|\lambda_{s}^{m}-\sqrt{-1}\langle k, \omega\rangle\right| \geq \frac{\alpha_{m}}{\Delta^{4}(|k|) \Delta^{4}([k])}
$$

and

$$
\left|\lambda_{s}^{m}-\lambda_{j}^{m}-\sqrt{-1}\langle k, \omega\rangle\right| \geq \frac{\alpha_{m}}{\Delta^{4}(|k|) \Delta^{4}([k])}
$$

for most $\varepsilon \in\left(0, \varepsilon_{0}\right)$ hold, where $1 \leq s, j \leq 2 N, m=0,1,2, \ldots, k \in \mathbf{Z}^{\mathbf{N}} \backslash\{0\}$, and $\Delta(t)$ is an approximation function.

Consider the Lipschitz constants from below and above of $f(\varepsilon)$ are $l(f(\varepsilon))$ and $L(f(\varepsilon))$, respectively. For any loss of generality, we can suppose that $\lambda_{s}^{m}-\lambda_{j}^{m}$ are pure imaginary numbers. So, we suppose

$$
\left|\lambda_{s}^{m}-\lambda_{j}^{m}-\sqrt{-1}\langle k, \omega\rangle\right| \geq \frac{\alpha_{m}}{\Delta^{4}(|k|) \Delta^{4}([k])}
$$

where $\lambda_{s}^{m}(\varepsilon)-\lambda_{j}^{m}(\varepsilon)$ satisfies $l\left(\lambda_{s}^{m}(\varepsilon)-\lambda_{j}^{m}(\varepsilon)\right) \geq \eta_{0}>0$ for a constant $\eta_{0}$ with $s \neq j$.

Remark Assume that $A_{m}(\varepsilon)$ is a Lipschitz function of $\varepsilon$ and $L\left(A_{m}\right)-L\left(A_{1}\right)=O(\varepsilon)$. By using Lemmas 3.9 and 3.10, and hypothesis (3) of Theorem 2.1, it is easy to prove that the eigenvalues $\lambda_{s}^{m}$ and the differences $\lambda_{s}^{m}-\lambda_{j}^{m}$ are Lipschitz from below and above if $\varepsilon$ is small enough.

The proof of the above remark can be seen in [5].

By condition (2) of Theorem 2.1, for $m=0$, equation (58) holds. And, by condition (2) of Theorem 2.1, we obtain that (58) also holds for $s=j$. Let

$$
f(\varepsilon)=\lambda_{s}^{m}-\lambda_{j}^{m}-\sqrt{-1}\langle k, \omega\rangle, \quad s \neq j
$$

and

$$
O_{s j m}^{k}=\left\{\varepsilon \in\left(0, \varepsilon_{0}\right):|f(\varepsilon)|<\frac{\alpha_{m}}{\Delta^{4}(|k|) \Delta^{4}([k])}\right\},
$$


such that system (30) converges for $\varepsilon \in\left(0, \varepsilon_{0}\right)$ whenever $\varepsilon_{0}$ is small enough and

$$
l\left(\lambda_{s}^{m}(\varepsilon)-\lambda_{j}^{m+1}(\varepsilon)\right) \mid \geq \eta_{0} .
$$

Since

$$
\begin{aligned}
|f(\varepsilon)| & \geq\left|\lambda_{s}-\lambda_{j}-\sqrt{-1}\langle k, \omega\rangle\right|-2 M \varepsilon \\
& \geq \frac{\alpha_{0}}{\Delta(|k|) \Delta([k])}-2 M \varepsilon_{0} .
\end{aligned}
$$

For $|k| \leq m,[k] \leq m$, and if $\frac{1}{4 \Delta(|k|) \Delta([k])}>\frac{M \varepsilon_{0}}{\alpha_{0}}$, then

$$
\begin{aligned}
|f(\varepsilon)| & \geq \frac{\alpha_{0}}{\Delta(|k|) \Delta([k])}-\frac{\alpha_{0}}{2 \Delta(m) \Delta(m)} \\
& \geq \frac{\alpha_{0}}{\Delta^{4}(|k|) \Delta^{4}([k])} \\
& \geq \frac{\alpha_{m}}{\Delta^{4}(|k|) \Delta^{4}([k])}
\end{aligned}
$$

and $O_{s j m}^{k}=\phi$.

Let, for $|k| \geq m,[k] \geq m$, and if $\frac{1}{4 \Delta(|k|) \Delta([k])}<\frac{\eta \varepsilon_{0}}{\alpha_{0}}$. By equation (59), we have

$$
\begin{aligned}
\operatorname{mes}\left(O_{s j m}^{k}\right)<\frac{\alpha_{m}}{\Delta^{4}(|k|) \Delta^{4}([k]) \eta_{0}}, & \\
\operatorname{mes}\left(\bigcup_{s \neq j} \bigcup_{k \in \mathbf{Z}^{\mathbb{N}} \backslash\{0\}} O_{s j m}^{k}\right) & \leq \sum_{1 \leq s, j \leq 2 d, k:|k| \geq m,[k] \geq m} \operatorname{mes}\left(O_{s j m}^{k}\right) \\
& \leq \frac{c d^{2} \alpha_{m} \varepsilon_{0}}{\alpha \Delta^{3}(m)} \sum_{k \in \mathbf{Z}^{\mathbb{N} \backslash\{0\}}} \frac{1}{\Delta(|k|) \Delta[k]} \\
& \leq \frac{c \varepsilon_{0}^{2}}{\Delta^{3}(m)} .
\end{aligned}
$$

Let

$$
E_{m}=\left\{\varepsilon \in\left(0, \varepsilon_{0}\right):\left|\lambda_{s}^{m}-\lambda_{j}^{m}-\sqrt{-1}\langle k, \omega\rangle\right| \geq \frac{\alpha_{m}}{\Delta^{4}(|k|) \Delta^{4}([k])}, k \in \mathbf{Z}^{\mathbf{N}} \backslash 0, s \neq j\right\} .
$$

Then

$$
\left(0, \varepsilon_{0}\right)-E_{m}=\bigcup_{s \neq j} \bigcup_{k \in \mathbf{Z}^{\mathbb{N}} \backslash\{0\}} O_{s j m}^{k}
$$

Thus

$$
\operatorname{mes}\left(\left(0, \varepsilon_{0}\right)-E_{m}\right)=\leq \frac{c \varepsilon_{0}^{2}}{\Delta^{3}(m)} .
$$

Let $E=\bigcap_{m=1}^{\infty} E_{m}$, then

$$
\operatorname{mes}\left(\left(0, \varepsilon_{0}\right)-E_{m}\right) \leq c \varepsilon_{0}^{2},
$$


and

$$
\lim _{\varepsilon_{0} \rightarrow 0} \frac{\operatorname{mes}\left(\left(0, \varepsilon_{0}\right)-E_{m}\right)}{\varepsilon_{0}}=0 .
$$

So, if $\varepsilon_{0}$ is sufficiently small, $E$ is a non-empty subset of $\left(0, \varepsilon_{0}\right)$. Similar to the above, for sufficiently small $\varepsilon_{0},\left|\lambda_{s}^{m}-\sqrt{-1}\langle k, \omega\rangle\right| \geq \frac{\alpha_{m}}{\Delta^{4}(|k|) \Delta^{4}([k])}$ holds for most $\varepsilon \in\left(0, \varepsilon_{0}\right)$. Hence,we proved that system (1) is changed into the Hamiltonian system (10).

\section{Conclusion}

In this work, we discussed the reducibility of almost-periodic Hamiltonian systems and proved that the almost-periodic non-linear Hamiltonian system (1) is reduced to a constant coefficients Hamiltonian system with an equilibrium by means of an almost-periodic symplectic transformation. The result was proved for a sufficiently small parameter $\varepsilon$ by using some non-resonant conditions, non-degeneracy conditions, the suitable hypothesis of analyticity, and KAM iterations.

\section{Acknowledgements}

The authors are grateful to Professor Daxiong Piao for his guidance and useful discussions

Funding

This work was supported by the NSFC (grant no. 11571327), NSF of Shandong Province (grant no. ZR2013AM026), and Chinese Scholarship Council, P.R. China (CSC No. 2014GXY552).

Competing interests

The authors declare that they have no competing interests.

\section{Authors' contributions}

All authors of this research paper have equally participated in the planning, execution, and analysis of this study. All authors read and approved the final manuscript.

\section{Author details}

'School of Mathematical Sciences, Ocean University of China, Qingdao, P.R. China. ${ }^{2}$ Department of Mathematics, Government College University, Lahore, Pakistan. ${ }^{3}$ Department of Mathematics and Statistics, Pir Mehr Ali Shah Arid Agriculture University, Rawalpindi, Pakistan.

\section{Publisher's Note}

Springer Nature remains neutral with regard to jurisdictional claims in published maps and institutional affiliations.

Received: 24 April 2018 Accepted: 18 July 2018 Published online: 28 July 2018

\section{References}

1. Palmer, K.J.: On the reducibility of almost-periodic systems of linear differential equations. J. Differ. Equ. 36, 374-390 (1980)

2. Johnson, R.A., Sell, G.R.: Smoothness of spectral subbundles and reducibility of quasiperodic linear differential systems. J. Differ. Equ. 41, 262-288 (1981)

3. Jorba, A., Simó, C.: On the reducibility of linear differential equations with quasi-periodic coefficients. J. Differ. Equ. 98, 111-124 (1992)

4. Xu, J., You, J.: On the reducibility of linear differential equations with almost-periodic coefficients. Chinese Ann. Math. A (in Chinese), 607-616 (1996)

5. Jorba, A., Simó, C.: On quasi-periodic perturbations of elliptic equilibrium points. SIAM J. Math. Anal. 27, 1704-1737 (1996)

6. Xu, J., You, J.: On the reducibility of a class of linear differential equations with quasi-periodic coefficients. Mathematika 46, 443-451 (1999)

7. Qiu, W., Si, J.: Reducibility for a class of almost-periodic differential equations with degenerate equilibrium point under small almost-periodic perturbations. Abstr. Appl. Anal. 2013, Article ID 386812 (2013)

8. Li, J., Zhu, C., Chen, S.: On the reducibility of a class of quasi-periodic Hamiltonian systems with small perturbation parameter near the equilibrium. Qual. Theory Dyn. Syst. 16, 127-147 (2015)

9. Pöschel, J.: Small divisors with spatial structure in infinite dimensional Hamiltonian systems. Commun. Math. Phys. 127, 351-393 (1990)

10. Rüssmann, R.: On the one-dimensional Schrödinger equation with a quasi-periodic potential. Ann. N.Y. Acad. Sci. 357, 90-107 (1980) 